\title{
Generating asymptotics for factorially divergent sequences
}

\author{
Michael Borinsky* \\ Departments of Physics and Mathematics \\ Humboldt-Universität zu Berlin \\ Berlin, Germany \\ borinsky@physik.hu-berlin.de
}

Submitted: Mar 9, 2016; Accepted: Jul 14, 2018; Published: Oct 5, 2018

(C) The author. Released under the CC BY license (International 4.0).

\begin{abstract}
The algebraic properties of formal power series, whose coefficients show factorial growth and admit a certain well-behaved asymptotic expansion, are discussed. It is shown that these series form a subring of $\mathbb{R}[[x]]$. This subring is also closed under composition and inversion of power series. An 'asymptotic derivation' is defined which maps a power series to the asymptotic expansion of its coefficients. Product and chain rules for this derivation are deduced. With these rules asymptotic expansions of the coefficients of implicitly defined power series can be obtained. The full asymptotic expansions of the number of connected chord diagrams and the number of simple permutations are given as examples.
\end{abstract}

Mathematics Subject Classifications: 05A16

\section{Introduction}

This article ${ }^{1}$ is concerned with real sequences $f_{n}$, which admit an asymptotic expansion for large $n$ of the form,

$$
f_{n}=\alpha^{n+\beta} \Gamma(n+\beta)\left(c_{0}+\frac{c_{1}}{\alpha(n+\beta-1)}+\frac{c_{2}}{\alpha^{2}(n+\beta-1)(n+\beta-2)}+\ldots\right),
$$

for some $\alpha \in \mathbb{R}_{>0}, \beta \in \mathbb{R}$ and $c_{k} \in \mathbb{R}$. Sequences of this type appear in many enumeration problems, which deal with coefficients of factorial growth. For instance, certain subclasses of permutations and graphs of fixed valence show this behaviour [1,8]. Furthermore,

*Supported by the German Academic Scholarship Foundation.

${ }^{1}$ An extended abstract of this article appeared as a contribution to FPSAC 2017 [12]. 
there are countless examples where perturbative expansions of physical quantities admit asymptotic expansions of this kind [5, 24, 17].

The restriction to this specific class of power series is inspired by the work of Bender. In [7] he analyzed the asymptotic behaviour of the coefficients of the composition of a power series, which has mildly growing coefficients, with a power series, which has rapidly growing coefficients. Here, Bender's results are extended into a complete algebraic framework. This is achieved by making heavy use of generating functions in the spirit of the 'analytic combinatorics' or 'symbolic method' approach $[20,10,28]$. The key step is to interpret the coefficients of the asymptotic expansion as another power series.

The resulting framework bears many resemblances to the theory of resurgence, which was established by Jean Ecalle [18]. Resurgence assigns a special role to power series whose coefficients grow factorially, as they offer themselves to be Borel transformed. For instance, it can be used to assign a unique function to such a factorially divergent sequence. This function could be interpreted as the sequence' generating function. Moreover, resurgence provides a promising approach to cope with divergent perturbative expansions in physics. Its application to these problems is an active field of research $[2,17,3]$.

During a conversation with David Sauzin it became plausible that the presented methods can also be derived from resurgence. In fact, the formalism can be seen as a toy model of resurgence's calcul différentiel étranger [18, Vol. 1] also called alien calculus [25, II.6]. This toy model is unable to fully reconstruct functions from asymptotic expansions, but does not rely on analytic properties of Borel transformed functions and therefore offers itself for combinatorial applications. A detailed and illuminating account on resurgence theory is given in Sauzin's review [25, Part II].

\section{$1.1 \quad$ Statement of results}

Power series whose coefficients have a well-behaved asymptotic expansion, as in eq. (1), form a subring of $\mathbb{R}[[x]]$, which will be denoted as $\mathbb{R}[[x]]_{\beta}^{\alpha}$. This subring is also closed under composition and inversion of power series. A linear map, $\mathcal{A}_{\beta}^{\alpha}: \mathbb{R}[[x]]_{\beta}^{\alpha} \rightarrow \mathbb{R}[[x]]$, can be defined which maps a power series to the asymptotic expansion of its coefficients. A natural way to define such a map is to associate the power series $\sum_{n=0}^{\infty} c_{n} x^{n}$ to the series $\sum_{n=0}^{\infty} f_{n} x^{n}$ related as in eq. (1). This map turns out to be a derivation that means it fulfills a product rule

with $f, g \in \mathbb{R}[[x]]_{\beta}^{\alpha} \quad\left(\mathcal{A}_{\beta}^{\alpha}(f \cdot g)\right)(x)=f(x)\left(\mathcal{A}_{\beta}^{\alpha} g\right)(x)+g(x)\left(\mathcal{A}_{\beta}^{\alpha} f\right)(x)$

and a chain rule, $\quad\left(\mathcal{A}_{\beta}^{\alpha}(f \circ g)\right)(x)=f^{\prime}(g(x))\left(\mathcal{A}_{\beta}^{\alpha} g\right)(x)+\left(\frac{x}{g(x)}\right)^{\beta} e^{\frac{1}{x}-\frac{1}{g(x)}}\left(\mathcal{A}_{\beta}^{\alpha} f\right)(g(x))$,

where $(f \cdot g)(x)=f(x) g(x)$ and $(f \circ g)(x)=f(g(x))$. In the second line it is required that $g_{0}=0$ and $g_{1}=1$. These statements will be derived from elementary properties of the $\Gamma$ function.

Note that the chain rule involves a peculiar correction term if the coefficients $f_{n}$ of the power series $f(x)$ have a non-trivial asymptotic expansion. It is obvious that the chain rule cannot be as simple as the ordinary chain rule for differentiation. For general $f, g \in$ 
$\mathbb{R}\left[[x]_{\beta}^{\alpha}:\left(\mathcal{A}_{\beta}^{\alpha}(f \circ g)\right)(x) \neq f^{\prime}(g(x))\left(\mathcal{A}_{\beta}^{\alpha} g\right)(x)\right.$. Otherwise, the reasonable requirement that the coefficients of the generating function $g(x)=x$ have a trivial asymptotic expansion, $\left(\mathcal{A}_{\beta}^{\alpha} g\right)(x)=0$, would imply that all $f \in \mathbb{R}[[x]]_{\beta}^{\alpha}$ have trivial asymptotic expansions.

In Sections 2-6 the derivation ring $\mathbb{R}[[x]]_{\beta}^{\alpha}$ will be described in detail and the main Theorem 35, which establishes the chain rule for the asymptotic derivation, will be proven. The formalism can be applied to calculate the asymptotic expansions of the coefficients of implicitly defined power series. This procedure is similar to the extraction of the derivative of an implicitly defined function using the implicit function theorem. We will use it in Section 7 to give the full asymptotic expansions of the number of connected chord diagrams and the full asymptotic expansions of the number of simple permutations.

\subsection{Notation}

A (formal) power series $f \in \mathbb{R}[[x]]$ will be denoted in the usual 'functional' notation $f(x)=\sum_{n=0}^{\infty} f_{n} x^{n}$. The coefficients of a power series $f$ will be expressed by the same symbol with the index attached as a subscript $f_{n}$ or with the coefficient extraction operator $\left[x^{n}\right] f(x)=f_{n}$. Ordinary (formal) derivatives are expressed as $f^{\prime}(x)=\sum_{n=0}^{\infty} n f_{n} x^{n-1}$. The (Cauchy) product of two power series $f, g$ will be expressed either as $f \cdot g,(f \cdot g)(x)$ or $f(x) g(x)$ depending on the context. Correspondingly, we will switch freely between the different notations $f \circ g,(f \circ g)(x)$ and $f(g(x))$ for the composition of two power series. The ring of power series, restricted to expansions of functions which are analytic at the origin, or equivalently power series with non-vanishing radius of convergence, will be denoted as $\mathbb{R}\{x\}$. The $\mathcal{O}$-notation will be used: $\mathcal{O}\left(a_{n}\right)$ denotes the set of all sequences $b_{n}$ such that $\limsup _{n \rightarrow \infty}\left|\frac{b_{n}}{a_{n}}\right|<\infty$ and $o\left(a_{n}\right)$ denotes all sequences $b_{n}$ such that $\lim _{n \rightarrow \infty} \frac{b_{n}}{a_{n}}=0$. Equations of the form $a_{n}=b_{n}+\mathcal{O}\left(c_{n}\right)$ are to be interpreted as statements $a_{n}-b_{n} \in \mathcal{O}\left(c_{n}\right)$ as usual. See [6] for an introduction to this notation. Tuples of non-negative integers will be denoted by bold letters $\mathbf{t}=\left(t_{1}, \ldots, t_{L}\right) \in \mathbb{N}_{0}^{L}$. The notation $|\mathbf{t}|$ will be used as a short form for $\sum_{l=1}^{L} t_{l}$. We will consider the binomial coefficient $\left(\begin{array}{l}a \\ n\end{array}\right)$ to be defined for all $a \in \mathbb{R}$ and $n \in \mathbb{N}_{0}$ by $\left(\begin{array}{l}a \\ n\end{array}\right):=\left[x^{n}\right](1+x)^{a}$.

The only non-standard notation that will be used to improve the readability of lengthy expressions is the abbreviation $\Gamma_{\beta}^{\alpha}(n):=\alpha^{n+\beta} \Gamma(n+\beta)$.

\section{Prerequisites}

We will start by defining the subset of power series whose coefficients have well-behaved asymptotic expansions:

Definition 1. For given $\alpha \in \mathbb{R}_{>0}$ and $\beta \in \mathbb{R}$ let $\mathbb{R}[[x]]_{\beta}^{\alpha}$ be the subset of $\mathbb{R}[[x]]$, such that $f \in \mathbb{R}[[x]]_{\beta}^{\alpha}$ if and only if there exists a sequence of real numbers $\left(c_{k}^{f}\right)_{k \in \mathbb{N}_{0}}$, which fulfills

$$
f_{n}=\sum_{k=0}^{R-1} c_{k}^{f} \Gamma_{\beta}^{\alpha}(n-k)+\mathcal{O}\left(\Gamma_{\beta}^{\alpha}(n-R)\right) \quad \forall R \in \mathbb{N}_{0},
$$

where $\Gamma_{\beta}^{\alpha}(n)=\alpha^{n+\beta} \Gamma(n+\beta)$. 
Observation 2. $\mathbb{R}[[x]]_{\beta}^{\alpha}$ is a linear subspace of $\mathbb{R}[[x]]$.

Observation 3. The sequence $\left(c_{k}^{f}\right)_{k \in \mathbb{N}_{0}}$ is unique for each fixed $f \in \mathbb{R}[[x]]_{\beta}^{\alpha}$. The coefficients can be calculated iteratively using the explicit formula $c_{K}^{f}=\lim _{n \rightarrow \infty} \frac{f_{n}-\sum_{k=0}^{K-1} c_{k}^{f} \Gamma_{\beta}^{\alpha}(n-k)}{\Gamma_{\beta}^{\alpha}(n-K)}$ for all $K \in \mathbb{N}_{0}$.

Both these properties follow immediately from Definition 1.

Remark 4. The expression in eq. (2) represents an asymptotic expansion or Poincaré expansion with the asymptotic scale $\alpha^{n+\beta} \Gamma(n+\beta)$ [16, Ch. 1.5].

Remark 5. The subspace $\mathbb{R}[[x]]_{\beta}^{\alpha}$ includes all (real) power series whose coefficients only grow exponentially: $\mathbb{R}\{x\} \subset \mathbb{R}[[x]]_{\beta}^{\alpha}$. These with all other series with coefficients, which are in $o\left(\Gamma_{\beta}^{\alpha}(n-R)\right)$ for all $R \in \mathbb{N}_{0}$, have an asymptotic expansion of the form in eq. (2) with all $c_{k}^{f}=0$.

Remark 6. Definition 1 implies that if $f \in \mathbb{R}[[x]]_{\beta}^{\alpha}$, then

$$
f_{n} \in \mathcal{O}\left(\Gamma_{\beta}^{\alpha}(n)\right)=\mathcal{O}\left(\alpha^{n} \Gamma(n+\beta)\right) .
$$

Accordingly, the power series in $\mathbb{R}[[x]]_{\beta}^{\alpha}$ are a subset of Gevrey-1 sequences [22, Ch XI2]. Being Gevrey-1 is not sufficient for a power series to be in $\mathbb{R}\left[[x]_{\beta}^{\alpha}\right.$. For instance, a sequence which behaves for large $n$ as $f_{n}=n !\left(1+\frac{1}{\sqrt{n}}+\mathcal{O}\left(\frac{1}{n}\right)\right)$ is Gevrey-1, but not in $\mathbb{R}[[x]]_{\beta}^{\alpha}$ for any pair $(\alpha, \beta)$.

Remark 7. In resurgence theory further restrictions on the allowed power series are imposed, which ensure that the Borel transformations of the sequences have proper analytic continuations or are 'endless continuable' [25, II.6]. These restrictions are analogous to the requirement that, apart from $f_{n}$, also the sequence $c_{k}^{f}$ has to have a well-behaved asymptotic expansion. The coefficients of this asymptotic expansion are also required to have a well-behaved asymptotic expansion and so on. These kinds of restrictions will not be necessary for the presented algebraic considerations, which are aimed at combinatorial applications.

The central theme of this article is to interpret the coefficients $c_{k}^{f}$ of the asymptotic expansion as another power series. In fact, Definition 1 immediately suggests to define the following map:

Definition 8. Let $\mathcal{A}_{\beta}^{\alpha}: \mathbb{R}[[x]]_{\beta}^{\alpha} \rightarrow \mathbb{R}[[x]]$ be the map that associates a power series $\mathcal{A}_{\beta}^{\alpha} f \in \mathbb{R}[[x]]$ to every power series $f \in \mathbb{R}[[x]]_{\beta}^{\alpha}$ such that

$$
\left(\mathcal{A}_{\beta}^{\alpha} f\right)(x)=\sum_{k=0}^{\infty} c_{k}^{f} x^{k},
$$

with the coefficients $c_{k}^{f}$ from Definition 1 .

Observation 9. $\mathcal{A}_{\beta}^{\alpha}$ is linear. 
Remark 10. In Proposition 22 it will be proven that $\mathcal{A}_{\beta}^{\alpha}$ is a derivation. We will adopt the usual notation for derivations and consider $\mathcal{A}_{\beta}^{\alpha}$ to act on everything to its right.

Remark 11. In the realm of resurgence such an operator is called alien derivative or alien operator [25, II.6].

Remark 12 . $\mathcal{A}_{\beta}^{\alpha}$ is clearly not injective. For instance, $\mathbb{R}\{x\} \subset \operatorname{ker} \mathcal{A}_{\beta}^{\alpha}$.

Example 13. The power series $f \in \mathbb{R}[[x]]$ associated to the sequence $f_{n}=n$ ! clearly fulfills the requirements of Definition 1 with $\alpha=1$ and $\beta=1$. Therefore, $f \in \mathbb{R}[[x]]_{1}^{1}$ and $\left(\mathcal{A}_{1}^{1} f\right)(x)=1$.

The asymptotic expansion in eq. (2) is normalized such that shifts in $k, c_{k}^{f} \rightarrow c_{k-m}^{f}$, can be absorbed by shifts in $\beta, \beta \rightarrow \beta+m$. More specifically,

Proposition 14. For all $m \in \mathbb{N}_{0}$

$$
f \in \mathbb{R}[[x]]_{\beta}^{\alpha} \text { if and only if } f \in \mathbb{R}[[x]]_{\beta+m}^{\alpha} \text { and } \mathcal{A}_{\beta+m}^{\alpha} f \in x^{m} \mathbb{R}[[x]] .
$$

If either holds, then $x^{m}\left(\mathcal{A}_{\beta}^{\alpha} f\right)(x)=\left(\mathcal{A}_{\beta+m}^{\alpha} f\right)(x)$.

Proof. Because $\Gamma_{\beta}^{\alpha}(n)=\alpha^{n-m+\beta+m} \Gamma(n-m+\beta+m)=\Gamma_{\beta+m}^{\alpha}(n-m)$, the following two relations between $f_{n}$ and $c_{k}^{f}$ are equivalent,

$$
\begin{aligned}
f_{n}=\sum_{k=0}^{R-1} c_{k}^{f} \Gamma_{\beta}^{\alpha}(n-k)+\mathcal{O}\left(\Gamma_{\beta}^{\alpha}(n-R)\right) & \forall R \in \mathbb{N}_{0} \\
f_{n}=\sum_{k=m}^{R^{\prime}-1} c_{k-m}^{f} \Gamma_{\beta+m}^{\alpha}(n-k)+\mathcal{O}\left(\Gamma_{\beta+m}^{\alpha}\left(n-R^{\prime}\right)\right) & \forall R^{\prime} \geqslant m .
\end{aligned}
$$

Eq. (4) follows from $f \in \mathbb{R}[[x]]_{\beta}^{\alpha}$ by Definition 1. In that case, eq. (5) implies that $f \in \mathbb{R}[[x]]_{\beta+m}^{\alpha}$ and that $\left(\mathcal{A}_{\beta+m}^{\alpha} f\right)(x)=\sum_{k=m}^{\infty} c_{k-m}^{f} x^{k}=x^{m}\left(\mathcal{A}_{\beta}^{\alpha} f\right)(x) \in x^{m} \mathbb{R}[[x]]$ by Definition 8.

If $f \in \mathbb{R}[[x]]_{\beta+m}^{\alpha}$ and $\mathcal{A}_{\beta+m}^{\alpha} f \in x^{m} \mathbb{R}[[x]]$, then we can write the asymptotic expansion of $f$ in the form of eq. (5). Eq. (4) implies $f \in \mathbb{R}[[x]]_{\beta}^{\alpha}$.

By analogous reasoning, we can absorb shifts in $n, f_{n} \rightarrow f_{n+m}$, in eq. (2) by shifts in $\beta, \beta \rightarrow \beta+m$.

Proposition 15. For all $m \in \mathbb{N}_{0}$

$$
f \in \mathbb{R}[[x]]_{\beta}^{\alpha} \cap x^{m} \mathbb{R}[[x]] \text { if and only if } \frac{f(x)}{x^{m}} \in \mathbb{R}[[x]]_{\beta+m}^{\alpha} .
$$

If either holds, then $\left(\mathcal{A}_{\beta}^{\alpha} f\right)(x)=\left(\mathcal{A}_{\beta+m}^{\alpha} \frac{f(x)}{x^{m}}\right)(x)$. 
Proof. Because $\Gamma_{\beta}^{\alpha}(n+m)=\alpha^{n+m+\beta} \Gamma(n+m+\beta)=\Gamma_{\beta+m}^{\alpha}(n)$, the following two relations between $f_{n}$ and $c_{k}^{f}$ are equivalent,

$$
\begin{array}{rlrl}
f_{n} & =\sum_{k=0}^{R-1} c_{k}^{f} \Gamma_{\beta}^{\alpha}(n-k)+\mathcal{O}\left(\Gamma_{\beta}^{\alpha}(n-R)\right) & \forall R \in \mathbb{N}_{0} \\
f_{n+m}=\sum_{k=0}^{R-1} c_{k}^{f} \Gamma_{\beta+m}^{\alpha}(n-k)+\mathcal{O}\left(\Gamma_{\beta+m}^{\alpha}(n-R)\right) & \forall R \in \mathbb{N}_{0} .
\end{array}
$$

Eq. (6) follows from $f \in \mathbb{R}[[x]]_{\beta}^{\alpha}$. Because $f \in x^{m} \mathbb{R}[[x]]$, we have $\frac{f(x)}{x^{m}}=\sum_{n=0}^{\infty} f_{n+m} x^{n} \in$ $\mathbb{R}[[x]]$. Eq. (7) then implies that $\frac{f(x)}{x^{m}} \in \mathbb{R}[[x]]_{\beta+m}^{\alpha}$ and by Definition $8,\left(\mathcal{A}_{\beta}^{\alpha} f\right)(x)=$ $\left(\mathcal{A}_{\beta+m}^{\alpha} \frac{f(x)}{x^{m}}\right)(x)$.

If $\frac{f(x)}{x^{m}} \in \mathbb{R}[[x]]_{\beta+m}^{\alpha} \subset \mathbb{R}[[x]]$, then $f \in x^{m} \mathbb{R}[[x]]$ and eq. (7) holds for the coefficients of $f$, which implies $f \in \mathbb{R}[[x]]_{\beta}^{\alpha}$ by eq. (6) and Definition 1 .

From Proposition 14, it follows that $\mathbb{R}[[x]]_{\beta}^{\alpha} \subset \mathbb{R}[[x]]_{\beta+m}^{\alpha}$ for all $m \in \mathbb{N}_{0}$. It will be convenient to only work in the spaces $\mathbb{R}[[x]]_{\beta}^{\alpha}$ with $\beta>0$ and to use Proposition 14 to verify that the subspaces $\mathbb{R}[[x]]_{\beta-m}^{\alpha}$ inherit all relevant properties from $\mathbb{R}[[x]]_{\beta}^{\alpha}$. The advantage is that, with $\beta>0$, it is easier to express uniform bounds on the remainder terms in eq. (2). The following definition will provide a convenient notation for these bounds.

Definition 16. For $\alpha, \beta \in \mathbb{R}_{>0}$ and $R \in \mathbb{N}_{0}$, let $\rho_{\beta, R}^{\alpha}: \mathbb{R}[[x]]_{\beta}^{\alpha} \rightarrow \mathbb{R}_{+}$be the map

$$
\rho_{\beta, R}^{\alpha}(f)=\max _{0 \leqslant K \leqslant R} \sup _{n \geqslant K} \frac{\left|f_{n}-\sum_{k=0}^{K-1} c_{k}^{f} \Gamma_{\beta}^{\alpha}(n-k)\right|}{\Gamma_{\beta}^{\alpha}(n-K)},
$$

with the coefficients $c_{k}^{f}$ as in Definition 1 .

It follows directly from Definition 1 that the quantity $\rho_{\beta, R}^{\alpha}(f)$ is finite. Eq. (8) can be translated into bounds for the coefficients $f_{n}$ and the $c_{k}^{f}$ :

Observation 17. If $\alpha, \beta \in \mathbb{R}_{>0}$ and $R \in \mathbb{N}_{0}$, then for all $f \in \mathbb{R}[[x]]_{\beta}^{\alpha}$ and $n, K \in \mathbb{N}_{0}$ with $K \leqslant R$ as well as $n \geqslant K$,

$$
\left|f_{n}-\sum_{k=0}^{K-1} c_{k}^{f} \Gamma_{\beta}^{\alpha}(n-k)\right| \leqslant \rho_{\beta, R}^{\alpha}(f) \Gamma_{\beta}^{\alpha}(n-K) \quad \text { and } \quad\left|c_{K}^{f}\right| \leqslant \rho_{\beta, R}^{\alpha}(f) .
$$

Remark 18. It can be verified using linearity and the triangle inequality that the maps $\rho_{\beta, R}^{\alpha}$ form a family of norms on all spaces $\mathbb{R}[[x]]_{\beta}^{\alpha}$ where $\beta>0$. Moreover, these norms will turn out to be submultiplicative up to equivalence (see Proposition 26). However, we will not make direct use of any topological properties of the spaces $\mathbb{R}[[x]]_{\beta}^{\alpha}$ in this article. 


\section{Elementary properties of sums over $\Gamma$ functions}

The following lemma forms the foundation for most of the conclusions that will follow. It provides an estimate for sums of $\Gamma$ functions. Moreover, it ensures that the subspace $\mathbb{R}[[x]]_{\beta}^{\alpha}$ of formal power series corresponds to a subset of a large class of sequences studied by Bender [7]. From another perspective the lemma can be seen as an entry point to resurgence, which bypasses the necessity for analytic continuations and Borel transformations.

Lemma 19. If $\alpha, \beta \in \mathbb{R}_{>0}$, then

$$
\sum_{m=0}^{n} \Gamma_{\beta}^{\alpha}(m) \Gamma_{\beta}^{\alpha}(n-m) \leqslant(2+\beta) \Gamma_{\beta}^{\alpha}(0) \Gamma_{\beta}^{\alpha}(n) \quad \forall n \in \mathbb{N}_{0} .
$$

Proof. Recall that $\Gamma_{\beta}^{\alpha}(n)=\alpha^{n+\beta} \Gamma(n+\beta)$ and that $\Gamma: \mathbb{R}_{>0} \rightarrow \mathbb{R}_{>0}$ is a log-convex function. If $\beta \in \mathbb{R}_{>0}$, then the functions $\Gamma(m+\beta)$ and $\Gamma(n-m+\beta)$ are also logconvex functions in $m$ on the interval $[0, n]$, as log-convexity is preserved under shifts and reflections. Furthermore, log-convexity is closed under multiplication. This implies that $\Gamma_{\beta}^{\alpha}(m) \Gamma_{\beta}^{\alpha}(n-m)=\alpha^{n+2 \beta} \Gamma(m+\beta) \Gamma(n-m+\beta)$ is a log-convex function in $m$ on the interval $[1, n-1] \subset[0, n]$. A convex function always attains its maximum on the boundary of its domain. Accordingly, $\Gamma_{\beta}^{\alpha}(m) \Gamma_{\beta}^{\alpha}(n-m) \leqslant \Gamma_{\beta}^{\alpha}(1) \Gamma_{\beta}^{\alpha}(n-1)$ for all $m \in[1, n-1]$. This way, the sum $\sum_{m=0}^{n} \Gamma_{\beta}^{\alpha}(m) \Gamma_{\beta}^{\alpha}(n-m)$ can be estimated after stripping off the two boundary terms:

$$
\sum_{m=0}^{n} \Gamma_{\beta}^{\alpha}(m) \Gamma_{\beta}^{\alpha}(n-m) \leqslant 2 \Gamma_{\beta}^{\alpha}(0) \Gamma_{\beta}^{\alpha}(n)+(n-1) \Gamma_{\beta}^{\alpha}(1) \Gamma_{\beta}^{\alpha}(n-1) \quad \forall n \geqslant 1 .
$$

It follows from $n \Gamma(n)=\Gamma(n+1)$ that $\Gamma_{\beta}^{\alpha}(1) \Gamma_{\beta}^{\alpha}(n-1)=\frac{\beta}{n-1+\beta} \Gamma_{\beta}^{\alpha}(0) \Gamma_{\beta}^{\alpha}(n)$ for all $n \geqslant 1$. Because $n-1+\beta \geqslant n-1$, substituting this into eq. (11) implies the inequality in eq. (10) for all $n \geqslant 1$. The remaining case $n=0$ is trivially fulfilled.

Corollary 20. If $\alpha, \beta \in \mathbb{R}_{>0}$ and $R \in \mathbb{N}_{0}$ are kept fixed, then there exists a constant $C \in \mathbb{R}$ such that

$$
\sum_{m=R}^{n-R} \Gamma_{\beta}^{\alpha}(m) \Gamma_{\beta}^{\alpha}(n-m) \leqslant C \Gamma_{\beta}^{\alpha}(n-R) \quad \forall n \geqslant 2 R .
$$

Proof. Recall that $\Gamma_{\beta}^{\alpha}(m+R)=\Gamma_{\beta+R}^{\alpha}(m)$. We can shift the summation variable to rewrite the left hand side of eq. (12) as

$$
\begin{gathered}
\sum_{m=0}^{n-2 R} \Gamma_{\beta}^{\alpha}(m+R) \Gamma_{\beta}^{\alpha}(n-m-R)=\sum_{m=0}^{n-2 R} \Gamma_{\beta+R}^{\alpha}(m) \Gamma_{\beta+R}^{\alpha}(n-2 R-m) \\
\leqslant(2+\beta+R) \Gamma_{\beta+R}^{\alpha}(0) \Gamma_{\beta+R}^{\alpha}(n-2 R),
\end{gathered}
$$

where we applied Lemma 19 with the substitutions $\beta \rightarrow \beta+R$ and $n \rightarrow n-2 R$. Because $\Gamma_{\beta+R}^{\alpha}(n-2 R)=\Gamma_{\beta}^{\alpha}(n-R)$ the statement follows. 
Corollary 21. If $\alpha, \beta \in \mathbb{R}_{>0}, C \in \mathbb{R}$ and $P \in \mathbb{R}[m]$ is some polynomial in $m$, then

$$
\sum_{m=R}^{n} C^{m} P(m) \Gamma_{\beta}^{\alpha}(n-m) \in \mathcal{O}\left(\Gamma_{\beta}^{\alpha}(n-R)\right) \quad \forall R \in \mathbb{N}_{0} .
$$

Proof. There is a constant $C^{\prime} \in \mathbb{R}$ such that $\left|C^{m} P(m)\right|$ is bounded by $C^{\prime} \Gamma_{\beta}^{\alpha}(m)$ for all $m \in \mathbb{N}_{0}$. Therefore, Corollary 20 ensures that

$$
\sum_{m=R}^{n-R} C^{m} P(m) \Gamma_{\beta}^{\alpha}(n-m) \leqslant C^{\prime} \sum_{m=R}^{n-R} \Gamma_{\beta}^{\alpha}(m) \Gamma_{\beta}^{\alpha}(n-m) \in \mathcal{O}\left(\Gamma_{\beta}^{\alpha}(n-R)\right) .
$$

The remainder $\sum_{m=n-R+1}^{n} C^{m} P(m) \Gamma_{\beta}^{\alpha}(n-m)=\sum_{m=0}^{R-1} C^{n-m} P(n-m) \Gamma_{\beta}^{\alpha}(m)$ is obviously in $\mathcal{O}\left(\Gamma_{\beta}^{\alpha}(n-R)\right)$.

\section{A derivation for asymptotics}

Proposition 22. If $\alpha \in \mathbb{R}_{>0}, \beta \in \mathbb{R}$ and $f, g \in \mathbb{R}[[x]]_{\beta}^{\alpha}$, then

- The product $(f \cdot g)(x)=f(x) g(x)$ belongs to $\mathbb{R}[[x]]_{\beta}^{\alpha}$.

- $\mathcal{A}_{\beta}^{\alpha}$ is a derivation, that means it respects the product rule

$$
\left(\mathcal{A}_{\beta}^{\alpha}(f \cdot g)\right)(x)=f(x)\left(\mathcal{A}_{\beta}^{\alpha} g\right)(x)+g(x)\left(\mathcal{A}_{\beta}^{\alpha} f\right)(x) .
$$

Corollary 23. If $g^{1}, \ldots, g^{L} \in \mathbb{R}[[x]]_{\beta}^{\alpha}$, then $\prod_{l=1}^{L} g^{l}(x) \in \mathbb{R}[[x]]_{\beta}^{\alpha}$ and

$$
\left(\mathcal{A}_{\beta}^{\alpha}\left(\prod_{l=1}^{L} g^{l}(x)\right)\right)(x)=\sum_{l=1}^{L}\left(\prod_{\substack{m=1 \\ m \neq l}}^{L} g^{m}(x)\right)\left(\mathcal{A}_{\beta}^{\alpha} g^{l}\right)(x)
$$

Proof. Proof by induction in $L$ using the product rule.

Corollary 24. If $g^{1}, \ldots, g^{L} \in \mathbb{R}[[x]]_{\beta}^{\alpha}$ and $\boldsymbol{t}=\left(t_{1}, \ldots, t_{L}\right) \in \mathbb{N}_{0}^{L}$, then $\prod_{l=1}^{L}\left(g^{l}(x)\right)^{t_{l}} \in$ $\mathbb{R}[[x]]_{\beta}^{\alpha}$ and

$$
\left(\mathcal{A}_{\beta}^{\alpha}\left(\prod_{l=1}^{L}\left(g^{l}(x)\right)^{t_{l}}\right)\right)(x)=\sum_{l=1}^{L} t_{l}\left(g^{l}(x)\right)^{t_{l}-1}\left(\prod_{\substack{m=1 \\ m \neq l}}^{L}\left(g^{m}(x)\right)^{t_{m}}\right)\left(\mathcal{A}_{\beta}^{\alpha} g^{l}\right)(x) .
$$

Corollary 25. If $g^{1}, \ldots, g^{L} \in \mathbb{R}[[x]]_{\beta}^{\alpha}$ and $p \in \mathbb{R}\left[y_{1}, \ldots, y_{L}\right]$ is polynomial in $L$ variables, then $p\left(g^{1}(x), \ldots, g^{L}(x)\right) \in \mathbb{R}[[x]]_{\beta}^{\alpha}$ and

$$
\left(\mathcal{A}_{\beta}^{\alpha}\left(p\left(g^{1}, \ldots, g^{L}\right)\right)\right)(x)=\sum_{l=1}^{L} \frac{\partial p}{\partial y_{l}}\left(g^{1}, \ldots, g^{L}\right)\left(\mathcal{A}_{\beta}^{\alpha} g^{l}\right)(x) .
$$


Although the last three statements are only basic general properties of commutative derivation rings, they suggest that $\mathcal{A}_{\beta}^{\alpha}$ fulfills a simple chain rule. In fact, Corollary 25 can still be generalized from polynomials to analytic functions (as we will do in Theorem 32), but, as already mentioned, this intuition turns out to be false in general.

We will prove Proposition 22 alongside with another statement which will be useful to establish the chain rule:

Proposition 26. If $\alpha, \beta \in \mathbb{R}_{>0}$ and $R \in \mathbb{N}_{0}$ are kept fixed, then there exists a constant $C \in \mathbb{R}$ such that

$$
\rho_{\beta, R}^{\alpha}(f \cdot g) \leqslant C \rho_{\beta, R}^{\alpha}(f) \rho_{\beta, R}^{\alpha}(g) \quad \forall f, g \in \mathbb{R}[[x]]_{\beta}^{\alpha} .
$$

Corollary 27. If $\alpha, \beta \in \mathbb{R}_{>0}, R \in \mathbb{N}_{0}$ and $g^{1}, \ldots, g^{L} \in \mathbb{R}[[x]]_{\beta}^{\alpha}$ are kept fixed, then there exists a constant $C \in \mathbb{R}$ such that

$$
\rho_{\beta, R}^{\alpha}\left(\prod_{l=1}^{L}\left(g^{l}(x)\right)^{t_{l}}\right) \leqslant C^{|\boldsymbol{t}|} \quad \forall \boldsymbol{t} \in \mathbb{N}_{0}^{L} \text { with }|\boldsymbol{t}| \geqslant 1 .
$$

Proof. Iterating eq. (18) gives a constant $C \in \mathbb{R}$ such that

$$
\rho_{\beta, R}^{\alpha}\left(\prod_{l=1}^{L}\left(g^{l}(x)\right)^{t_{l}}\right) \leqslant C^{|\mathbf{t}|-1} \prod_{l=1}^{L}\left(\rho_{\beta, R}^{\alpha}\left(g^{l}\right)\right)^{t_{l}} \quad \forall \mathbf{t} \in \mathbb{N}_{0}^{L} \text { with }|\mathbf{t}| \geqslant 1 .
$$

The right hand side is clearly bounded by $C^{\prime|\mathbf{t}|}$ for all $|\mathbf{t}| \geqslant 1$ with an appropriate $C^{\prime} \in \mathbb{R}$ which depends on the $g^{l}$.

We will prove Proposition 22 under the assumption that $\beta>0$. The following lemma shows that, as a consequence of Proposition 14, we can do so without loss of generality.

Lemma 28. If Proposition 22 holds for $\beta \in \mathbb{R}_{>0}$, then it holds for all $\beta \in \mathbb{R}$.

Proof. For $\beta \in \mathbb{R}$, choose $m \in \mathbb{N}_{0}$ such that $\beta+m>0$. If $f, g \in \mathbb{R}[[x]]_{\beta}^{\alpha}$, then $f, g \in \mathbb{R}[[x]]_{\beta+m}^{\alpha}$ by Proposition 14. By the requirement $f \cdot g \in \mathbb{R}[[x]]_{\beta+m}^{\alpha}$ and $\left(\mathcal{A}_{\beta+m}^{\alpha}(f\right.$. $g))(x)=f(x)\left(\mathcal{A}_{\beta+m}^{\alpha} g\right)(x)+g(x)\left(\mathcal{A}_{\beta+m}^{\alpha} f\right)(x)$. Using $\left(\mathcal{A}_{\beta+m}^{\alpha} f\right)(x)=x^{m}\left(\mathcal{A}_{\beta}^{\alpha} f\right)(x)$ from Proposition 14 gives $\left(\mathcal{A}_{\beta+m}^{\alpha}(f \cdot g)\right)(x)=x^{m}\left(f(x)\left(\mathcal{A}_{\beta}^{\alpha} g\right)(x)+g(x)\left(\mathcal{A}_{\beta}^{\alpha} f\right)(x)\right)$. Because $f \cdot g \in \mathbb{R}\left[[x]_{\beta+m}^{\alpha}\right.$ and $\mathcal{A}_{\beta+m}^{\alpha}(f \cdot g) \in x^{m} \mathbb{R}[[x]]$, it follows that $f \cdot g \in \mathbb{R}[[x]]_{\beta}^{\alpha}$ and $\mathcal{A}_{\beta}^{\alpha}(f \cdot g)=f(x)\left(\mathcal{A}_{\beta}^{\alpha} g\right)(x)+g(x)\left(\mathcal{A}_{\beta}^{\alpha} f\right)(x)$ by Proposition 14.

To prove Propositions 22 and 26, we will use some estimates for the coefficients of the product of two power series. To establish these estimates, we will require that $\beta>0$.

Lemma 29. If $\alpha, \beta \in \mathbb{R}_{>0}$ and $R \in \mathbb{N}_{0}$ are kept fixed, then there exists a constant $C \in \mathbb{R}$ such that for all $f, g \in \mathbb{R}[[x]]_{\beta}^{\alpha}$ and $n, K \in \mathbb{N}_{0}$ with $K \leqslant R$ as well as $n \geqslant K$,

$$
\left|\sum_{m=0}^{n} f_{n-m} g_{m}-\sum_{m=0}^{K-1} f_{n-m} g_{m}-\sum_{m=0}^{K-1} f_{m} g_{n-m}\right| \leqslant C \rho_{\beta, R}^{\alpha}(f) \rho_{\beta, R}^{\alpha}(g) \Gamma_{\beta}^{\alpha}(n-K) .
$$


Proof. Observation 17 with $K=0$ states that $\left|f_{n}\right| \leqslant \rho_{\beta, R}^{\alpha}(f) \Gamma_{\beta}^{\alpha}(n)$ for all $f \in \mathbb{R}[[x]]_{\beta}^{\alpha}$ and $n \in \mathbb{N}_{0}$. We can use this to estimate the expression

$$
h_{n}:=\left|\sum_{m=0}^{n} f_{n-m} g_{m}-\sum_{m=0}^{K-1} f_{n-m} g_{m}-\sum_{m=0}^{K-1} f_{m} g_{n-m}\right|
$$

in different ranges for $n$,

$$
\begin{aligned}
2 K>n \geqslant K \Rightarrow h_{n} & =\left|\sum_{m=n-K+1}^{K-1} f_{n-m} g_{m}\right| \leqslant \rho_{\beta, R}^{\alpha}(f) \rho_{\beta, R}^{\alpha}(g) \sum_{m=n-K+1}^{K-1} \Gamma_{\beta}^{\alpha}(n-m) \Gamma_{\beta}^{\alpha}(m) \\
n \geqslant 2 K \Rightarrow h_{n} & =\left|\sum_{m=K}^{n-K} f_{n-m} g_{m}\right| \leqslant \rho_{\beta, R}^{\alpha}(f) \rho_{\beta, R}^{\alpha}(g) \sum_{m=K}^{n-K} \Gamma_{\beta}^{\alpha}(n-m) \Gamma_{\beta}^{\alpha}(m) .
\end{aligned}
$$

It is trivial to find a constant $C$ such that $\sum_{m=n-K+1}^{K-1} \Gamma_{\beta}^{\alpha}(n-m) \Gamma_{\beta}^{\alpha}(m) \leqslant C \Gamma_{\beta}^{\alpha}(n-K)$ for all $K \leqslant R$ and $2 K>n \geqslant K$, because $R$ is fixed and only finitely many inequalities need to be fulfilled. Corollary 20 guarantees that we can also find a constant $C$ for the second case.

Lemma 30. If $\alpha, \beta \in \mathbb{R}_{>0}$ and $R \in \mathbb{N}_{0}$ are kept fixed, then there exists a constant $C \in \mathbb{R}$ such that for all $f, g \in \mathbb{R}[[x]]_{\beta}^{\alpha}$ and $n, K \in \mathbb{N}_{0}$ with $K \leqslant R$ as well as $n \geqslant K$,

$$
\left|\sum_{m=0}^{K-1} f_{n-m} g_{m}-\sum_{k=0}^{K-1} d_{k}^{f, g} \Gamma_{\beta}^{\alpha}(n-k)\right| \leqslant C \rho_{\beta, R}^{\alpha}(f) \rho_{\beta, R}^{\alpha}(g) \Gamma_{\beta}^{\alpha}(n-K),
$$

where $d_{k}^{f, g}:=\left[x^{k}\right] g(x)\left(\mathcal{A}_{\beta}^{\alpha} f\right)(x)$.

Proof. Observation 17 with the substitutions $n \rightarrow n-m$ and $K \rightarrow K-m$ implies that

$$
\left|f_{n-m}-\sum_{k=0}^{K-m-1} c_{k}^{f} \Gamma_{\beta}^{\alpha}(n-m-k)\right| \leqslant \rho_{\beta, R}^{\alpha}(f) \Gamma_{\beta}^{\alpha}(n-K),
$$

for all $f \in \mathbb{R}[[x]]_{\beta}^{\alpha}$ and $n, K, m \in \mathbb{N}_{0}$ with $m \leqslant K \leqslant R$ as well as $n \geqslant K$ where $c_{k}^{f}=\left[x^{k}\right]\left(\mathcal{A}_{\beta}^{\alpha} f\right)(x)$. It also follows from Observation 17 that $\left|g_{m}\right| \leqslant \rho_{\beta, R}^{\alpha}(g) \Gamma_{\beta}^{\alpha}(m)$ for all $g \in \mathbb{R}\left[[x]_{\beta}^{\alpha}\right.$ and $m \in \mathbb{N}_{0}$. Because $d_{k}^{f, g}=\sum_{m=0}^{k} c_{k-m}^{f} g_{m}$,

$$
\begin{gathered}
\left|\sum_{m=0}^{K-1} f_{n-m} g_{m}-\sum_{k=0}^{K-1} d_{k}^{f, g} \Gamma_{\beta}^{\alpha}(n-k)\right|=\left|\sum_{m=0}^{K-1} f_{n-m} g_{m}-\sum_{k=0}^{K-1} \sum_{m=0}^{k} c_{k-m}^{f} g_{m} \Gamma_{\beta}^{\alpha}(n-k)\right| \\
=\left|\sum_{m=0}^{K-1}\left(f_{n-m}-\sum_{k=m}^{K-1} c_{k-m}^{f} \Gamma_{\beta}^{\alpha}(n-k)\right) g_{m}\right| \leqslant \sum_{m=0}^{K-1}\left|f_{n-m}-\sum_{k=0}^{K-m-1} c_{k}^{f} \Gamma_{\beta}^{\alpha}(n-m-k)\right|\left|g_{m}\right| \\
\leqslant \rho_{\beta, R}^{\alpha}(f) \rho_{\beta, R}^{\alpha}(g) \Gamma_{\beta}^{\alpha}(n-K) \sum_{m=0}^{K-1} \Gamma_{\beta}^{\alpha}(m) \quad \forall n \geqslant K .
\end{gathered}
$$

Setting $C=\sum_{m=0}^{R-1} \Gamma_{\beta}^{\alpha}(m)$ results in the statement. 
Lemma 31. If $\alpha, \beta \in \mathbb{R}_{>0}$ and $R \in \mathbb{N}_{0}$ are kept fixed, then there exists a constant $C \in \mathbb{R}$ such that for all $f, g \in \mathbb{R}[[x]]_{\beta}^{\alpha}$ and $n, K \in \mathbb{N}_{0}$ with $K \leqslant R$ as well as $n \geqslant K$,

$$
\left|\sum_{m=0}^{n} f_{n-m} g_{m}-\sum_{k=0}^{K-1} c_{k}^{f \cdot g} \Gamma_{\beta}^{\alpha}(n-k)\right| \leqslant C \rho_{\beta, R}^{\alpha}(f) \rho_{\beta, R}^{\alpha}(g) \Gamma_{\beta}^{\alpha}(n-K),
$$

where $c_{k}^{f \cdot g}:=\left[x^{k}\right]\left(f(x)\left(\mathcal{A}_{\beta}^{\alpha} g\right)(x)+g(x)\left(\mathcal{A}_{\beta}^{\alpha} f\right)(x)\right)$.

Proof. Note that $c_{k}^{f \cdot g}=d_{k}^{f, g}+d_{k}^{g, f}$ with $d_{k}^{f, g}$ from Lemma 30 and $d_{k}^{g, f}$ respectively with the roles of $f$ and $g$ switched. We can use the triangle inequality to deduce that

$$
\begin{gathered}
\left|\sum_{m=0}^{n} f_{n-m} g_{m}-\sum_{k=0}^{K-1} c_{k}^{f \cdot g} \Gamma_{\beta}^{\alpha}(n-k)\right| \leqslant\left|\sum_{m=0}^{n} f_{n-m} g_{m}-\sum_{m=0}^{K-1} f_{n-m} g_{m}-\sum_{m=0}^{K-1} f_{m} g_{n-m}\right| \\
\quad+\left|\sum_{m=0}^{K-1} f_{n-m} g_{m}-\sum_{k=0}^{K-1} d_{k}^{f, g} \Gamma_{\beta}^{\alpha}(n-k)\right|+\left|\sum_{m=0}^{K-1} f_{m} g_{n-m}-\sum_{k=0}^{K-1} d_{k}^{g, f} \Gamma_{\beta}^{\alpha}(n-k)\right| .
\end{gathered}
$$

Using Lemmas 29 and 30 on the respective terms on the right hand side of this inequality results in the statement.

Proof of Proposition 22. By Lemma 28, it is sufficient to prove Proposition 22 for $\beta>0$. Therefore, we can apply Lemma 31 for $f, g \in \mathbb{R}[[x]]_{\beta}^{\alpha}$. Eq. (22) with $K=R$ directly implies that

$$
\left[x^{n}\right] f(x) g(x)=\sum_{m=0}^{n} f_{n-m} g_{m}=\sum_{k=0}^{R-1} c_{k}^{f \cdot g} \Gamma_{\beta}^{\alpha}(n-k)+\mathcal{O}\left(\Gamma_{\beta}^{\alpha}(n-R)\right) \quad \forall R \in \mathbb{N}_{0},
$$

with $c_{k}^{f \cdot g}=\left[x^{k}\right]\left(f(x)\left(\mathcal{A}_{\beta}^{\alpha} g\right)(x)+g(x)\left(\mathcal{A}_{\beta}^{\alpha} f\right)(x)\right)$. By Definition 1, it follows that $f \cdot g \in$ $\mathbb{R}[[x]]_{\beta}^{\alpha}$ and from Definition 8 follows eq. (14).

Proof of Proposition 26. If $f, g \in \mathbb{R}[[x]]_{\beta}^{\alpha}$, then $f \cdot g \in \mathbb{R}[[x]]_{\beta}^{\alpha}$ by Proposition 22. Because $\beta>0$, we have by Definition 16

$$
\rho_{\beta, R}^{\alpha}(f \cdot g)=\max _{0 \leqslant K \leqslant R} \sup _{n \geqslant K} \frac{\left|\sum_{m=0}^{n} f_{n-m} g_{m}-\sum_{k=0}^{K-1} c_{k}^{f \cdot g} \Gamma_{\beta}^{\alpha}(n-k)\right|}{\Gamma_{\beta}^{\alpha}(n-K)} \quad \forall f, g \in \mathbb{R}[[x]]_{\beta}^{\alpha},
$$

which is bounded by $C \rho_{\beta, R}^{\alpha}(f) \rho_{\beta, R}^{\alpha}(g)$ with some fixed $C \in \mathbb{R}$ as follows directly from Lemma 31.

\section{Composition}

\subsection{Composition by analytic functions}

Theorem 32. If $\alpha \in \mathbb{R}_{>0}, \beta \in \mathbb{R}, f \in \mathbb{R}\left\{y_{1}, \ldots, y_{L}\right\}$ is a function in $L$ variables, which is analytic at the origin, and $g^{1}, \ldots, g^{L} \in \mathbb{R}[[x]]_{\beta}^{\alpha} \cap x \mathbb{R}[[x]]$, then 
- The composition $f\left(g^{1}(x), \ldots, g^{L}(x)\right)$ is in $\mathbb{R}\left[[x]_{\beta}^{\alpha}\right.$.

- $\mathcal{A}_{\beta}^{\alpha}$ fulfills a multivariate chain rule for the composition with analytic functions,

$$
\left(\mathcal{A}_{\beta}^{\alpha} f\left(g^{1}, \ldots, g^{L}\right)\right)(x)=\sum_{l=1}^{L} \frac{\partial f}{\partial y_{l}}\left(g^{1}, \ldots, g^{L}\right)\left(\mathcal{A}_{\beta}^{\alpha} g^{l}\right)(x) .
$$

In [7] Edward Bender established this theorem for the case $L=1$ in a less 'generatingfunctionology' based notation. If, for example, $g \in \mathbb{R}\left[[x]_{\beta}^{\alpha}\right.$ and $f \in \mathbb{R}\{x, y\}$, then his Theorem 1 allows us to calculate the asymptotic expansion of the coefficients of the power series $f(g(x), x)$. In fact, Bender analyzed more general power series including series with coefficients which grow even more rapidly than factorially.

The following proof of Theorem 32 is a straightforward generalization of Bender's Lemma 2 and Theorem 1 in [7] to the multivariate case $f \in \mathbb{R}\left\{y_{1}, \ldots, y_{L}\right\}$.

Again, we will start by verifying that we may assume $\beta>0$ during the proof of Theorem 23.

Lemma 33. If Theorem 32 holds for $\beta \in \mathbb{R}_{>0}$, then it also holds for all $\beta \in \mathbb{R}$.

Proof. For $\beta \in \mathbb{R}$, choose an $m \in \mathbb{N}_{0}$ such that $\beta+m>0$. If $g^{1}, \ldots, g^{L} \in \mathbb{R}[[x]]_{\beta}^{\alpha} \cap x \mathbb{R}[[x]]$, then by Proposition $14, g^{1}, \ldots, g^{L} \in \mathbb{R}[[x]]_{\beta+m}^{\alpha} \cap x \mathbb{R}[[x]],\left(\mathcal{A}_{\beta+m}^{\alpha} g^{l}\right)(x)=x^{m}\left(\mathcal{A}_{\beta}^{\alpha} g^{l}\right)(x)$ and by the requirement $h(x):=f\left(g^{1}(x), \ldots, g^{L}(x)\right) \in \mathbb{R}\left[[x]_{\beta+m}^{\alpha}\right.$ as well as

$$
\left(\mathcal{A}_{\beta+m}^{\alpha} h\right)(x)=\sum_{l=1}^{L} \frac{\partial f}{\partial y_{l}}\left(g^{1}, \ldots, g^{L}\right)\left(\mathcal{A}_{\beta+m}^{\alpha} g^{l}\right)(x)=x^{m} \sum_{l=1}^{L} \frac{\partial f}{\partial y_{l}}\left(g^{1}, \ldots, g^{L}\right)\left(\mathcal{A}_{\beta}^{\alpha} g^{l}\right)(x) .
$$

Due to Proposition 14, $h \in \mathbb{R}[[x]]_{\beta}^{\alpha}$ and $\left(\mathcal{A}_{\beta}^{\alpha} h\right)(x)=\sum_{l=1}^{L} \frac{\partial f}{\partial y_{l}}\left(g^{1}, \ldots, g^{L}\right)\left(\mathcal{A}_{\beta}^{\alpha} g^{l}\right)(x)$.

As before, we will use our freedom to assume that $\beta>0$ to establish an estimate on the coefficients of products of power series in $x \mathbb{R}[[x]]_{\beta}^{\alpha}$.

Lemma 34. If $\alpha, \beta \in \mathbb{R}_{>0}$ and $g^{1}, \ldots, g^{L} \in \mathbb{R}[[x]]_{\beta}^{\alpha} \cap x \mathbb{R}[[x]]$ are kept fixed, then there exists a constant $C \in \mathbb{R}$ such that

$$
\left|\left[x^{n}\right] \prod_{l=1}^{L}\left(g^{l}(x)\right)^{t_{l}}\right| \leqslant C^{|t|} \Gamma_{\beta}^{\alpha}(n-|\boldsymbol{t}|+1) \quad \forall \boldsymbol{t} \in \mathbb{N}_{0}^{L}, n \in \mathbb{N}_{0} \text { with } n \geqslant|\boldsymbol{t}| \geqslant 1 .
$$

Proof. By Proposition 15, it follows from $g^{l} \in \mathbb{R}[[x]]_{\beta}^{\alpha} \cap x \mathbb{R}[[x]]$ that $\frac{g^{l}(x)}{x} \in \mathbb{R}[[x]]_{\beta+1}^{\alpha}$ and therefore by Corollary 24 that $\prod_{l=1}^{L}\left(\frac{g^{l}(x)}{x}\right)^{t_{l}} \in \mathbb{R}[[x]]_{\beta+1}^{\alpha}$ for all $\mathbf{t} \in \mathbb{N}_{0}^{L}$. We can apply Observation 17 with $R=K=0$ to obtain for all $n \geqslant|\mathbf{t}|$,

$$
\left|\left[x^{n}\right] \prod_{l=1}^{L}\left(g^{l}(x)\right)^{t_{l}}\right|=\left|\left[x^{n-|\mathbf{t}|}\right] \prod_{l=1}^{L}\left(\frac{g^{l}(x)}{x}\right)^{t_{l}}\right| \leqslant \rho_{\beta+1,0}^{\alpha}\left(\prod_{l=1}^{L}\left(\frac{g^{l}(x)}{x}\right)^{t_{l}}\right) \Gamma_{\beta+1}^{\alpha}(n-|\mathbf{t}|) .
$$

The statement follows from Corollary 27 and $\Gamma_{\beta+1}^{\alpha}(n-|\mathbf{t}|)=\Gamma_{\beta}^{\alpha}(n-|\mathbf{t}|+1)$. 
Proof of Theorem 32. The composition $f\left(g^{1}(x), \ldots, g^{L}(x)\right)$ can be expressed as the sum $\sum_{\mathbf{t} \in \mathbb{N}_{0}^{L}} f_{t_{1}, \ldots, t_{L}} \prod_{l=1}^{L}\left(g^{l}(x)\right)^{t_{l}}$, which can be split in preparation for the extraction of the coefficients and their asymptotics:

$$
f\left(g^{1}(x), \ldots, g^{L}(x)\right)=\sum_{\substack{\mathbf{t} \in \mathbb{N}_{0}^{L} \\|\mathbf{t}| \leqslant R}} f_{t_{1}, \ldots, t_{L}} \prod_{l=1}^{L}\left(g^{l}(x)\right)^{t_{l}}+\sum_{\substack{\mathbf{t} \in \mathbb{N}_{0}^{L} \\|\mathbf{t}|>R}} f_{t_{1}, \ldots, t_{L}} \prod_{l=1}^{L}\left(g^{l}(x)\right)^{t_{l}} \quad \forall R \in \mathbb{N}_{0} .
$$

The first sum is just the composition by a polynomial. Corollary 25 asserts that this sum is in $\mathbb{R}[[x]]_{\beta}^{\alpha}$. It has the asymptotic expansion given in eq. (17) which agrees, as a series in $x$, with the right hand side of eq. (23) up to order $R-1$, because the partial derivative reduces the order of a polynomial by one and $g_{0}^{l}=0$.

It is left to prove that the coefficients of the power series given by the remaining sum over $|\mathbf{t}|>R$ are in $\mathcal{O}\left(\Gamma_{\beta}^{\alpha}(n-R)\right)$. Because of Lemma 33, we may assume that $\beta>0$ without loss of generality and apply Lemma 34 . Together with the fact that there is a constant $C$, such that $\left|f_{t_{1}, \ldots, t_{L}}\right| \leqslant C^{|\mathbf{t}|}$ for all $\mathbf{t} \in \mathbb{N}_{0}^{L}$, due to the analyticity of $f$, Lemma 34 ensures that there is a constant $C^{\prime} \in \mathbb{R}$ such that

$$
\begin{gathered}
\left|\left[x^{n}\right] \sum_{\substack{\mathbf{t} \in \mathbb{N}_{0}^{L} \\
|\mathbf{t}|>R}} f_{t_{1}, \ldots, t_{L}} \prod_{l=1}^{L}\left(g^{l}(x)\right)^{t_{l}}\right| \leqslant \sum_{\substack{\mathbf{t} \in \mathbb{N}_{0}^{L} \\
n \geqslant|\mathbf{t}|>R}}\left|f_{t_{1}, \ldots, t_{L}}\right|\left|\left[x^{n}\right] \prod_{l=1}^{L}\left(g^{l}(x)\right)^{t_{l}}\right| \\
\leqslant \sum_{t=R+1}^{n} C^{\prime t} \Gamma_{\beta}^{\alpha}(n-t+1) \sum_{\substack{\mathbf{t} \in \mathbb{N}_{0}^{L} \\
|\mathbf{t}|=t}} 1,
\end{gathered}
$$

for all $n \geqslant R+1$. Because the last sum $\left|\left\{t_{1}, \ldots, t_{L} \in \mathbb{N}_{0} \mid t_{1}+\ldots+t_{L}=t\right\}\right|=\left(\begin{array}{c}t+L-1 \\ L-1\end{array}\right)$ is a polynomial in $t$, Corollary 21 asserts that this is in $\mathcal{O}\left(\Gamma_{\beta}^{\alpha}(n-R)\right)$.

\subsection{Proof of the main theorem: Composition of power series in $\mathbb{R}[[x]]_{\beta}^{\alpha}$}

Despite the fact that Bender's theorem applies to a broader range of compositions $f \circ g$, where $f$ does not need to be analytic and $g$ does not need to be an element of $\mathbb{R}[[x]]_{\beta}^{\alpha}$, it cannot be used in the case $f, g \in \mathbb{R}[[x]]_{\beta}^{\alpha}$, where $f \notin \operatorname{ker} \mathcal{A}_{\beta}^{\alpha}$. The problem is that we cannot truncate the sum $\sum_{k=0}^{\infty} f_{k} g(x)^{k}$ without losing significant information. In this section, we will confront this problem and prove the general chain rule for the asymptotic derivative:

Theorem 35. If $\alpha \in \mathbb{R}_{>0}, \beta \in \mathbb{R}$ and $f, g \in \mathbb{R}[[x]]_{\beta}^{\alpha}$ with $g_{0}=0$ and $g_{1}=1$, then

- The composition $f \circ g$ and the inverse $g^{-1}$ belong to $\mathbb{R}[[x]]_{\beta}^{\alpha}$. 
- $\mathcal{A}_{\beta}^{\alpha}$ fulfills a chain rule and there is a formula for the $\mathcal{A}_{\beta}^{\alpha}$-derivative of the compositional inverse:

$$
\begin{array}{r}
\left(\mathcal{A}_{\beta}^{\alpha}(f \circ g)\right)(x)=f^{\prime}(g(x))\left(\mathcal{A}_{\beta}^{\alpha} g\right)(x)+\left(\frac{x}{g(x)}\right)^{\beta} e^{\frac{\frac{1}{x}-\frac{1}{g(x)}}{\alpha}}\left(\mathcal{A}_{\beta}^{\alpha} f\right)(g(x)), \\
\left(\mathcal{A}_{\beta}^{\alpha} g^{-1}\right)(x)=-\left(g^{-1}\right)^{\prime}(x)\left(\frac{x}{g^{-1}(x)}\right)^{\beta} e^{\frac{\frac{1}{x}-\frac{1}{g^{-1}(x)}}{\alpha}}\left(\mathcal{A}_{\beta}^{\alpha} g\right)\left(g^{-1}(x)\right) .
\end{array}
$$

Corollary 36. If $f \in \mathbb{R}[[x]], g \in \mathbb{R}[[x]]_{\beta}^{\alpha}$ with $g_{0}=0, g_{1}=1$ and $f \circ g \in \mathbb{R}[[x]]_{\beta}^{\alpha}$, then $f \in \mathbb{R}[[x]]_{\beta}^{\alpha}$.

Proof. Theorem 35 guarantees that $g^{-1} \in \mathbb{R}[[x]]_{\beta}^{\alpha}$ and therefore also $f=(f \circ g) \circ g^{-1} \in$ $\mathbb{R}[[x]]_{\beta}^{\alpha}$.

As before, we will assume that $\beta>0$ while proving Theorem 35. The following lemma establishes that we can do so.

Lemma 37. If Theorem 35 holds for $\beta \in \mathbb{R}_{>0}$, then it holds for all $\beta \in \mathbb{R}$.

Proof. For $\beta \in \mathbb{R}$, choose $m \in \mathbb{N}_{0}$ such that $\beta+m>0$. If $f, g \in \mathbb{R}[[x]]_{\beta}^{\alpha}$ with $g_{0}=0, g_{1}=1$, then $f, g \in \mathbb{R}[[x]]_{\beta+m}^{\alpha}$ by Proposition 14. Because of $\left(\mathcal{A}_{\beta+m}^{\alpha} f\right)(x)=x^{m}\left(\mathcal{A}_{\beta}^{\alpha} f\right)(x)$ and by the requirement

$$
\begin{gathered}
\left(\mathcal{A}_{\beta+m}^{\alpha}(f \circ g)\right)(x)=f^{\prime}(g(x))\left(\mathcal{A}_{\beta+m}^{\alpha} g\right)(x)+\left(\frac{x}{g(x)}\right)^{\beta+m} e^{\frac{\frac{1}{x}-\frac{1}{g(x)}}{\alpha}}\left(\mathcal{A}_{\beta+m}^{\alpha} f\right)(g(x)) \\
=x^{m}\left(f^{\prime}(g(x))\left(\mathcal{A}_{\beta}^{\alpha} g\right)(x)+\left(\frac{x}{g(x)}\right)^{\beta} e^{\frac{\frac{1}{x}-\frac{1}{g(x)}}{\alpha}}\left(\mathcal{A}_{\beta}^{\alpha} f\right)(g(x))\right) .
\end{gathered}
$$

Applying Proposition 14 again results in $f \circ g \in \mathbb{R}[[x]]_{\beta}^{\alpha}$ and eq. (25). Eq. (26) and $g^{-1} \in \mathbb{R}[[x]]_{\beta}^{\alpha}$ follow analogously.

Obviously, $x \in \mathbb{R}[[x]]_{\beta}^{\alpha}$. We will use this basic fact to prove Theorem 35 by ensuring that from $f, g \in \mathbb{R}[[x]]_{\beta}^{\alpha}$ follows $f \circ g^{-1} \in \mathbb{R}[[x]]_{\beta}^{\alpha}$ and by constructing the asymptotic expansion of the coefficients of $\left(f \circ g^{-1}\right)(x)$. To prove that $f \circ g^{-1} \in \mathbb{R}[[x]]_{\beta}^{\alpha}$, the Lagrange inversion formula, which involves the usual derivative of a power series, will be used. To handle this derivative, the following proposition will become necessary:

Proposition 38. If $f \in \mathbb{R}[[x]]_{\beta}^{\alpha}$, then $f^{\prime}(x) \in \mathbb{R}[[x]]_{\beta+2}^{\alpha}$ and

$$
\left(\mathcal{A}_{\beta+2}^{\alpha} f^{\prime}\right)(x)=\left(\alpha^{-1}-x \beta+x^{2} \frac{\partial}{\partial x}\right)\left(\mathcal{A}_{\beta}^{\alpha} f\right)(x) .
$$

Proof. Recall that $f^{\prime}(x)=\sum_{n=0}^{\infty} n f_{n} x^{n-1}=\sum_{n=0}^{\infty}(n+1) f_{n+1} x^{n}$. If $f \in \mathbb{R}[[x]]_{\beta}^{\alpha}$, then by Definition 1,

$$
(n+1) f_{n+1}=\sum_{k=0}^{R-1} c_{k}^{f}(n+1) \Gamma_{\beta}^{\alpha}(n+1-k)+(n+1) \mathcal{O}\left(\Gamma_{\beta}^{\alpha}(n+1-R)\right) \quad \forall R \in \mathbb{N}_{0} .
$$


Observe that because $x \Gamma(x)=\Gamma(x+1)$ and $\Gamma_{\beta}^{\alpha}(n)=\alpha^{n+\beta} \Gamma(n+\beta)$,

$$
\begin{gathered}
(n+1) \Gamma_{\beta}^{\alpha}(n+1-k) \\
=\alpha^{n+1-k+\beta}((n+1-k+\beta) \Gamma(n+1-k+\beta)+(k-\beta) \Gamma(n+1-k+\beta)) \\
=\alpha^{-1} \Gamma_{\beta+2}^{\alpha}(n-k)+(k-\beta) \Gamma_{\beta+2}^{\alpha}(n-k-1) .
\end{gathered}
$$

Therefore, for all $R \in \mathbb{N}_{0}$

$$
(n+1) f_{n+1}=\sum_{k=0}^{R-1} c_{k}^{f}\left(\alpha^{-1} \Gamma_{\beta+2}^{\alpha}(n-k)+(k-\beta) \Gamma_{\beta+2}^{\alpha}(n-k-1)\right)+\mathcal{O}\left(\Gamma_{\beta+2}^{\alpha}(n-R)\right),
$$

and it follows from Definition 1 that $f^{\prime} \in \mathbb{R}[[x]]_{\beta+2}^{\alpha}$. Moreover, by Definition 8 ,

$$
\begin{aligned}
\left(\mathcal{A}_{\beta+2}^{\alpha} f^{\prime}\right)(x) & =\sum_{k=0}^{\infty} c_{k}^{f^{\prime}} x^{k}=\sum_{k=0}^{\infty} c_{k}^{f}\left(\alpha^{-1} x^{k}+(k-\beta) x^{k+1}\right) \\
= & \left(\alpha^{-1}-x \beta+x^{2} \frac{\partial}{\partial x}\right)\left(\mathcal{A}_{\beta}^{\alpha} f\right)(x) .
\end{aligned}
$$

While using the Lagrange inversion formula to establish $f \circ g^{-1} \in \mathbb{R}[[x]]_{\beta}^{\alpha}$, it will be convenient to work in the rings $\mathbb{R}[[x]]_{\beta+1}^{\alpha}$ and $\mathbb{R}[[x]]_{\beta+2}^{\alpha}$, which contain $\mathbb{R}[[x]]_{\beta}^{\alpha}$ as a subring. Therefore, we will start with some observations on intermediate quantities in $\mathbb{R}[[x]]_{\beta+1}^{\alpha}$ and $\mathbb{R}[[x]]_{\beta+2}^{\alpha}$. The following three lemmas are basic applications of the chain rule for the composition with analytic functions and the product rule, but we will prove them in detail to get acquainted to the new notions from the last sections.

Lemma 39. If $g \in \mathbb{R}[[x]]_{\beta}^{\alpha}$ with $g_{0}=0, g_{1}=1$ and $\gamma \in \mathbb{R}$, then $\left(\frac{g(x)}{x}\right)^{\gamma} \in \mathbb{R}[[x]]_{\beta+1}^{\alpha}$ and

$$
\left(\mathcal{A}_{\beta+1}^{\alpha}\left(\frac{g(x)}{x}\right)^{\gamma}\right)=\gamma\left(\frac{g(x)}{x}\right)^{\gamma-1}\left(\mathcal{A}_{\beta}^{\alpha} g\right)(x) .
$$

Proof. Observe that $F(x):=(1-x)^{\gamma} \in \mathbb{R}\{x\}$ and $F^{\prime}(x)=-\gamma(1-x)^{\gamma-1}$. Proposition 15 implies that $\frac{g(x)}{x} \in \mathbb{R}[[x]]_{\beta+1}^{\alpha}$, because $g \in \mathbb{R}[[x]]_{\beta}^{\alpha} \cap x \mathbb{R}[[x]]$. As $g_{1}=1$, we additionally have $1-\frac{g(x)}{x} \in \mathbb{R}[[x]]_{\beta+1}^{\alpha} \cap x \mathbb{R}[[x]]$. Using Theorem 32 results in

$$
\left(\frac{g(x)}{x}\right)^{\gamma}=F\left(1-\frac{g(x)}{x}\right) \in \mathbb{R}[[x]]_{\beta+1}^{\alpha}
$$

and by the chain rule for the composition with analytic functions from eq. (23),

$$
\begin{gathered}
\left(\mathcal{A}_{\beta+1}^{\alpha}\left(\frac{g(x)}{x}\right)^{\gamma}\right)=F^{\prime}\left(1-\frac{g(x)}{x}\right)\left(\mathcal{A}_{\beta+1}^{\alpha}\left(1-\frac{g(x)}{x}\right)\right)(x) \\
=-\gamma\left(\frac{g(x)}{x}\right)^{\gamma-1}\left(\mathcal{A}_{\beta+1}^{\alpha}\left(-\frac{g(x)}{x}\right)\right)(x)=\gamma\left(\frac{g(x)}{x}\right)^{\gamma-1}\left(\mathcal{A}_{\beta}^{\alpha} g\right)(x),
\end{gathered}
$$

where we used the linearity of $\mathcal{A}_{\beta+1}^{\alpha}$ and $\left(\mathcal{A}_{\beta+1}^{\alpha} \frac{g(x)}{x}\right)(x)=\left(\mathcal{A}_{\beta}^{\alpha} g\right)(x)$ due to Proposition 15 . 
Lemma 40. If $g \in \mathbb{R}[[x]]_{\beta}^{\alpha}$ with $g_{0}=0, g_{1}=1$, then

$$
\begin{gathered}
A(x):=\frac{1}{g(x)}-\frac{1}{x} \in \mathbb{R}[[x]]_{\beta+2}^{\alpha}, e^{\frac{A(x)}{\alpha}} \in \mathbb{R}[[x]]_{\beta+2}^{\alpha} \text { and } \\
\left(\mathcal{A}_{\beta+2}^{\alpha} e^{\frac{A(x)}{\alpha}}\right)(x)=-\alpha^{-1}\left(\frac{x}{g(x)}\right)^{2} e^{\frac{A(x)}{\alpha}}\left(\mathcal{A}_{\beta}^{\alpha} g\right)(x) .
\end{gathered}
$$

Proof. From Lemma 39 with $\gamma=-1$, it follows that $\frac{x}{g(x)} \in \mathbb{R}[[x]]_{\beta+1}^{\alpha}$ and

$$
\left(\mathcal{A}_{\beta+1}^{\alpha} \frac{x}{g(x)}\right)=-\left(\frac{x}{g(x)}\right)^{2}\left(\mathcal{A}_{\beta}^{\alpha} g\right)(x) .
$$

Because $g_{1}=1, \frac{x}{g(x)}-1 \in \mathbb{R}[[x]]_{\beta+1}^{\alpha} \cap x \mathbb{R}[[x]]$. Moreover, by Proposition $15, A(x)=$ $\frac{\frac{x}{g(x)}-1}{x} \in \mathbb{R}[[x]]_{\beta+2}^{\alpha}$ and

$$
\left(\mathcal{A}_{\beta+2}^{\alpha} A\right)(x)=\left(\mathcal{A}_{\beta+1}^{\alpha}\left(\frac{x}{g(x)}-1\right)\right)(x)=-\left(\frac{x}{g(x)}\right)^{2}\left(\mathcal{A}_{\beta}^{\alpha} g\right)(x) .
$$

Observe that $\frac{A(x)-A(0)}{\alpha} \in \mathbb{R}[[x]]_{\beta+2}^{\alpha} \cap x \mathbb{R}[[x]]$. Because $e^{x} \in \mathbb{R}\{x\}$, we can apply Theorem 32 to conclude that $e^{\frac{A(x)-A(0)}{\alpha}} \in \mathbb{R}[[x]]_{\beta+2}^{\alpha}$ and by linearity that also $e^{\frac{A(x)}{\alpha}} \in \mathbb{R}[[x]]_{\beta+2}^{\alpha}$. Finally, we can use the chain rule for the composition with analytic functions to write the left hand side of eq. (30) as

$$
\begin{gathered}
e^{\frac{A(0)}{\alpha}}\left(\mathcal{A}_{\beta+2}^{\alpha} e^{\frac{A(x)-A(0)}{\alpha}}\right)(x)=e^{\frac{A(0)}{\alpha}} e^{\frac{A(x)-A(0)}{\alpha}}\left(\mathcal{A}_{\beta+2}^{\alpha} \frac{A(x)-A(0)}{\alpha}\right)(x) \\
=e^{\frac{A(x)}{\alpha}}\left(\mathcal{A}_{\beta+2}^{\alpha} \frac{A(x)}{\alpha}\right)(x)=\alpha^{-1} e^{\frac{A(x)}{\alpha}}\left(\mathcal{A}_{\beta+2}^{\alpha} A\right)(x) .
\end{gathered}
$$

The statement in eq. (30) follows after substitution of $\left(\mathcal{A}_{\beta+2}^{\alpha} A\right)(x)$ from eq. (31).

Lemma 41. If $f, g \in \mathbb{R}[[x]]_{\beta}^{\alpha}$ with $g_{0}=0, g_{1}=1$ and $\gamma \in \mathbb{R}$, then

$$
\begin{gathered}
B_{\gamma}(x):=f(x) g^{\prime}(x)\left(\frac{g(x)}{x}\right)^{\gamma} \in \mathbb{R}[[x]]_{\beta+2}^{\alpha} \quad \text { and } \\
\left(\mathcal{A}_{\beta+2}^{\alpha} B_{\gamma}\right)(x)= \\
\left(\frac{g(x)}{x}\right)^{\gamma}\left(x^{2} g^{\prime}(x)\left(\mathcal{A}_{\beta}^{\alpha} f\right)(x)+f(x)\left(\gamma x g^{\prime}(x) \frac{x}{g(x)}+\alpha^{-1}-\beta x+x^{2} \frac{\partial}{\partial x}\right)\left(\mathcal{A}_{\beta}^{\alpha} g\right)(x)\right) .
\end{gathered}
$$

Proof. Recall that due to Proposition 14, $f \in \mathbb{R}[[x]]_{\beta}^{\alpha} \subset \mathbb{R}[[x]]_{\beta+2}^{\alpha}$ and $\left(\mathcal{A}_{\beta+2}^{\alpha} f\right)(x)=$ $x^{2}\left(\mathcal{A}_{\beta}^{\alpha} f\right)(x)$. Proposition 38 guarantees that $g^{\prime} \in \mathbb{R}[[x]]_{\beta+2}^{\alpha}$ and

$$
\left(\mathcal{A}_{\beta+2}^{\alpha} g^{\prime}\right)(x) f=\left(\alpha^{-1}-x \beta+x^{2} \frac{\partial}{\partial x}\right)\left(\mathcal{A}_{\beta}^{\alpha} g\right)(x) .
$$


Because of Lemma 39 and Proposition 14, we have $\left(\frac{g(x)}{x}\right)^{\gamma} \in \mathbb{R}[[x]]_{\beta+2}^{\alpha}$ and

$$
\left(\mathcal{A}_{\beta+2}^{\alpha}\left(\frac{g(x)}{x}\right)^{\gamma}\right)(x)=x \gamma\left(\frac{g(x)}{x}\right)^{\gamma-1}\left(\mathcal{A}_{\beta}^{\alpha} g\right)(x) .
$$

Putting all this together we can use Corollary 23 with $g^{1}(x)=f(x), g^{2}(x)=g^{\prime}(x)$ and $g^{3}(x)=\left(\frac{g(x)}{x}\right)^{\gamma}$ to obtain eqs. (32) and (33).

Lemma 42. If $\alpha, \beta \in \mathbb{R}_{>0}, R \in \mathbb{N}_{0}$ and $A, B_{\gamma}$ as defined in eqs. (29) and (32) are kept fixed, then there exists a constant $C \in \mathbb{R}$ such that

$$
\rho_{\beta+2, R}^{\alpha}\left(B_{\gamma}(x) A(x)^{m}\right) \leqslant C^{m+1} \quad \forall m \in \mathbb{N}_{0} .
$$

Proof. Apply Corollary 24 with $g^{1}(x)=B_{\gamma}(x), g^{2}(x)=A(x), t_{1}=1$ and $t_{2}=m$ to verify that $B_{\gamma}(x) A(x)^{m} \in \mathbb{R}[[x]]_{\beta+2}^{\alpha}$ for all $m \in \mathbb{N}_{0}$. Apply Corollary 27 with the same parameters.

Corollary 43. If $\alpha, \beta \in \mathbb{R}_{>0}, R \in \mathbb{N}_{0}$ and $A, B_{\gamma}$ as defined in eqs. (29) and (32) are kept fixed, then there exists a constant $C \in \mathbb{R}$ such that

$$
\left|\left[x^{n}\right] B_{\gamma}(x) A(x)^{m}-\sum_{k=0}^{R-1} c_{k, m} \Gamma_{\beta+2}^{\alpha}(n-k)\right| \leqslant C^{m+1} \Gamma_{\beta+2}^{\alpha}(n-R) \quad \forall n \geqslant R \text { and } m \in \mathbb{N}_{0}
$$

where $c_{k, m}=\left[x^{k}\right]\left(\mathcal{A}_{\beta+2}^{\alpha} B_{\gamma}(x) A(x)^{m}\right)(x)$.

Proof. Additionally to Lemma 42, apply Observation 17 with $K=R$.

The key to the extraction of the large $n$ asymptotics of $\left[x^{n}\right]\left(f \circ g^{-1}\right)(x)$ is a variant of the $\mathrm{Chu}$-Vandermonde identity. We will prove this identity using elementary power series techniques.

Lemma 44. For all $a \in \mathbb{R}$ and $m, k \in \mathbb{N}_{0}$

$$
\left(\begin{array}{c}
a \\
m
\end{array}\right)=\sum_{l=0}^{m}\left(\begin{array}{c}
k+l-1 \\
l
\end{array}\right)\left(\begin{array}{c}
a-k-l \\
m-l
\end{array}\right) .
$$

Proof. Recall that $\left(\begin{array}{l}a \\ n\end{array}\right)=\left[x^{n}\right](1+x)^{a}$ for all $a \in \mathbb{R}$ and $n \in \mathbb{N}_{0}$. By standard generating function arguments it follows that $\left[x^{n}\right] \frac{1}{(1-x)^{k}}=\left(\begin{array}{c}k+n-1 \\ n\end{array}\right)$ for all $n, k \in \mathbb{N}_{0}$. Observe that for all $a \in \mathbb{R}$ and $k \in \mathbb{N}_{0}$, we have the following identities in $\mathbb{R}[[x]]$ :

$$
\begin{gathered}
(1+x)^{a}=(1+x)^{k}(1+x)^{a-k}=\frac{1}{\left(1-\frac{x}{1+x}\right)^{k}}(1+x)^{a-k} \\
=\sum_{l=0}^{\infty}\left(\begin{array}{c}
k+l-1 \\
l
\end{array}\right)\left(\frac{x}{1+x}\right)^{l}(1+x)^{a-k}=\sum_{l=0}^{\infty}\left(\begin{array}{c}
k+l-1 \\
l
\end{array}\right) x^{l}(1+x)^{a-k-l} .
\end{gathered}
$$

Extracting coefficients from the first and the last expression results in the Chu-Vandermonde-type identity in eq. (35). 
Corollary 45. For all $\alpha, \beta \in \mathbb{R}_{>0}$ and $n, R, k \in \mathbb{N}_{0}$ with $n \geqslant R \geqslant k$, we have the identity in $\mathbb{R}[x]$

$$
\sum_{m=0}^{n-R}\left(\begin{array}{c}
n+\beta+1 \\
m
\end{array}\right) \Gamma_{\beta+2}^{\alpha}(n-m-k) x^{m}=\sum_{l=0}^{n-R}\left(\begin{array}{c}
l+k-1 \\
l
\end{array}\right) \Gamma_{\beta+2}^{\alpha}(n-l-k) x^{l} \sum_{m=0}^{n-R-l} \frac{\left(\frac{x}{\alpha}\right)^{m}}{m !} .
$$

Proof. Observe that $\left(\begin{array}{l}a \\ n\end{array}\right)=\frac{1}{n !} \frac{\Gamma(a+1)}{\Gamma(a-n+1)}$ for all $a \in \mathbb{R}$ and $n \in \mathbb{N}_{0}$ as long as $n<a+1$. By writing the second binomial coefficient on the right hand side of eq. (35) in this form and setting $a=n+\beta+1$, we get for all $n, m, k \in \mathbb{N}_{0}$ with $m+k<n+\beta+2$

$$
\left(\begin{array}{c}
n+\beta+1 \\
m
\end{array}\right) \Gamma(n-m-k+\beta+2)=\sum_{l=0}^{m}\left(\begin{array}{c}
k+l-1 \\
l
\end{array}\right) \frac{\Gamma(n-k-l+\beta+2)}{(m-l) !} .
$$

Multiplying by $x^{m} \alpha^{n-m-k+\beta+2}$, summing over $m$ and using $\Gamma_{\beta}^{\alpha}(n)=\alpha^{n+\beta} \Gamma(n+\beta)$ gives,

$$
\sum_{m=0}^{n-R}\left(\begin{array}{c}
n+\beta+1 \\
m
\end{array}\right) \Gamma_{\beta+2}^{\alpha}(n-m-k) x^{m}=\sum_{m=0}^{n-R} \sum_{l=0}^{m}\left(\begin{array}{c}
k+l-1 \\
l
\end{array}\right) \frac{\alpha^{l-m} \Gamma_{\beta+2}^{\alpha}(n-k-l)}{(m-l) !} x^{m} .
$$

Note that $k \leqslant R$ and $m \leqslant n-R$ imply $m+k \leqslant n<n+\beta+2$. The statement follows after changing the order of summation and a shift of the summation variable $m \rightarrow m+l$ both on the right hand side of this equation.

We are now equipped with the necessary tools to tackle the asymptotic analysis of the coefficients of $\left(f \circ g^{-1}\right)(x)$. The first step is to express $\left(f \circ g^{-1}\right)(x)$ in terms of the intermediate power series $A(x)$ and $B_{\gamma}(x)$. We will do so using a variant of the Lagrange inversion theorem.

Lemma 46. If $p, q \in \mathbb{R}[[x]]$ with $q_{0}=0$ and $q_{1}=1$, then

$$
\left[x^{n}\right] p\left(q^{-1}(x)\right)=\left[x^{n}\right] p(x) q^{\prime}(x)\left(\frac{x}{q(x)}\right)^{n+1} \quad \forall n \in \mathbb{N}_{0} .
$$

Proof. Note that the identity holds for $n=0$, because $q_{0}=0$ and $q_{1}=1$. It follows from the Lagrange inversion theorem [20, A.6] for $n \geqslant 1$ that

$$
\begin{gathered}
{\left[x^{n}\right] p\left(q^{-1}(x)\right)=\frac{1}{n}\left[x^{n-1}\right] p^{\prime}(x)\left(\frac{x}{q(x)}\right)^{n}} \\
=\frac{1}{n}\left[x^{n-1}\right] \frac{\partial}{\partial x}\left(p(x)\left(\frac{x}{q(x)}\right)^{n}\right)-\frac{1}{n}\left[x^{n-1}\right] p(x)\left(\frac{\partial}{\partial x}\left(\frac{x}{q(x)}\right)^{n}\right) .
\end{gathered}
$$

Using $\frac{1}{n}\left[x^{n-1}\right] \frac{\partial}{\partial x}=\left[x^{n}\right]$ and evaluating the derivative in the second term result in the statement. 
Corollary 47. If $\alpha, \beta \in \mathbb{R}_{>0}, f, g \in \mathbb{R}[[x]]_{\beta}^{\alpha}$ and $A, B_{\gamma}$ as defined in eqs. (29) and (32), then

$$
\left[x^{n}\right] f\left(g^{-1}(x)\right)=\sum_{m=0}^{n}\left(\begin{array}{c}
n+\beta+1 \\
m
\end{array}\right)\left[x^{n-m}\right] B_{\beta}(x) A(x)^{m} \quad \forall n \in \mathbb{N}_{0} .
$$

Proof. By Lemma 46,

$$
\left[x^{n}\right] f\left(g^{-1}(x)\right)=\left[x^{n}\right] f(x) g^{\prime}(x)\left(\frac{x}{g(x)}\right)^{n+1}=\left[x^{n}\right] f(x) g^{\prime}(x)\left(\frac{g(x)}{x}\right)^{\beta}\left(\frac{x}{g(x)}\right)^{n+\beta+1} .
$$

Using the definitions of $A$ and $B_{\gamma}$ gives $\left[x^{n}\right] f\left(g^{-1}(x)\right)=\left[x^{n}\right] B_{\beta}(x)(1+x A(x))^{n+\beta+1}$. Expanding with the generalized binomial theorem results in eq. (38).

Corollary 48. If $\alpha, \beta \in \mathbb{R}_{>0}, f, g \in \mathbb{R}[[x]]_{\beta}^{\alpha}$ and $A, B_{\gamma}$ as defined in eqs. (29) and (32), then

$$
\left[x^{n}\right] f\left(g^{-1}(x)\right)=\sum_{m=0}^{n-R}\left(\begin{array}{c}
n+\beta+1 \\
m
\end{array}\right)\left[x^{n-m}\right] B_{\beta}(x) A(x)^{m}+\mathcal{O}\left(\Gamma_{\beta+2}^{\alpha}(n-R)\right) \quad \forall R \in \mathbb{N}_{0} .
$$

Proof. Eq. (39) follows from eq. (38) and

$$
\begin{aligned}
\left|\sum_{m=n-R+1}^{n}\left(\begin{array}{c}
n+\beta+1 \\
m
\end{array}\right)\left[x^{n-m}\right] B_{\beta}(x) A(x)^{m}\right| & =\left|\sum_{m=0}^{R-1}\left(\begin{array}{c}
n+\beta+1 \\
n-m
\end{array}\right)\left[x^{m}\right] B_{\beta}(x) A(x)^{n-m}\right| \\
\leqslant \sum_{m=0}^{R-1}\left(\begin{array}{c}
n+\beta+1 \\
n-m
\end{array}\right) C^{n-m+1} \Gamma_{\beta+2}^{\alpha}(m) & \in \mathcal{O}\left(\Gamma_{\beta+2}^{\alpha}(n-R)\right) \quad \forall R \in \mathbb{N}_{0},
\end{aligned}
$$

where the second step, together with the existence of an appropriate $C \in \mathbb{R}$, follows from Corollary 43 with $R=0$ and the inclusion holds, because $\left(\begin{array}{c}n+\beta+1 \\ n-m\end{array}\right)=\frac{\Gamma(n+\beta+2)}{\Gamma(n-m+1) \Gamma(\beta+m+2)} \sim$ $\frac{n^{\beta+m+1}}{\Gamma(\beta+m+2)}$ by elementary properties of the $\Gamma$ function.

Lemma 49. If $\alpha, \beta \in \mathbb{R}_{>0}, f, g \in \mathbb{R}[[x]]_{\beta}^{\alpha}$ and $A, B_{\gamma}$ as defined in eqs. (29) and (32), then

$$
\begin{array}{r}
{\left[x^{n}\right] f\left(g^{-1}(x)\right)=\sum_{k=0}^{R-1} \sum_{l=0}^{n-R} \sum_{m=0}^{n-R-l} c_{k, l, m}\left(\begin{array}{c}
l+k-1 \\
l
\end{array}\right) \Gamma_{\beta+2}^{\alpha}(n-l-k)+\mathcal{O}\left(\Gamma_{\beta+2}^{\alpha}(n-R)\right)} \\
\forall R \in \mathbb{N}_{0},
\end{array}
$$

where $c_{k, l, m}:=\left[x^{k}\right]\left(\mathcal{A}_{\beta+2}^{\alpha} B_{\beta}(x) A(x)^{l} \frac{\left(\frac{A(x)}{\alpha}\right)^{m}}{m !}\right)(x)$.

Note that the terms of the triple sum in eq. (40) where $k=0$ are not all trivial, because $\left(\begin{array}{c}-1 \\ 0\end{array}\right)=1$ by the definition of the binomial coefficients with negative arguments. 
Proof. For all $n, m \in \mathbb{N}_{0}$ with $n-m \geqslant R$ set

$$
\mathcal{R}_{n, m}:=\left[x^{n-m}\right] B_{\beta}(x) A(x)^{m}-\sum_{k=0}^{R-1} c_{k, m} \Gamma_{\beta+2}^{\alpha}(n-m-k),
$$

where $c_{k, m}=\left[x^{k}\right]\left(\mathcal{A}_{\beta+2}^{\alpha} B_{\beta}(x) A(x)^{m}\right)(x)$. Substituting $\mathcal{R}_{n, m}$ into eq. (39) gives

$$
\begin{aligned}
& {\left[x^{n}\right] f\left(g^{-1}(x)\right)=\sum_{m=0}^{n-R}\left(\begin{array}{c}
n+\beta+1 \\
m
\end{array}\right) \sum_{k=0}^{R-1} c_{k, m} \Gamma_{\beta+2}^{\alpha}(n-m-k)} \\
& +\sum_{m=0}^{n-R}\left(\begin{array}{c}
n+\beta+1 \\
m
\end{array}\right) \mathcal{R}_{n, m}+\mathcal{O}\left(\Gamma_{\beta+2}^{\alpha}(n-R)\right) \quad \forall R \in \mathbb{N}_{0},
\end{aligned}
$$

By Corollary 43 with $n \rightarrow n-m$, we can find a constant $C \in \mathbb{R}$ such that $\left|\mathcal{R}_{n, m}\right| \leqslant$ $C^{m+1} \Gamma_{\beta+2}^{\alpha}(n-m-R)$ for all $n-m \geqslant R$. Therefore,

$$
\mathcal{R}_{n}:=\left|\sum_{m=0}^{n-R}\left(\begin{array}{c}
n+\beta+1 \\
m
\end{array}\right) \mathcal{R}_{n, m}\right| \leqslant \sum_{m=0}^{n-R}\left(\begin{array}{c}
n+\beta+1 \\
m
\end{array}\right) C^{m+1} \Gamma_{\beta+2}^{\alpha}(n-m-R) \quad \forall n \geqslant R .
$$

Applying Corollary 45 with $x \rightarrow C$ and $k=R$ results in

$$
\begin{aligned}
\mathcal{R}_{n} & \leqslant C \sum_{l=0}^{n-R}\left(\begin{array}{c}
l+R-1 \\
l
\end{array}\right) \Gamma_{\beta+2}^{\alpha}(n-l-R) C^{l} \sum_{m=0}^{n-R-l} \frac{\left(\frac{C}{\alpha}\right)^{m}}{m !} & & \forall n \geqslant R \\
& \leqslant C \sum_{l=R}^{n}\left(\begin{array}{l}
l-1 \\
l-R
\end{array}\right) \Gamma_{\beta+2}^{\alpha}(n-l) C^{l-R} \sum_{m=0}^{n-R} \frac{\left(\frac{C}{\alpha}\right)^{m}}{m !} & & \forall n \geqslant R .
\end{aligned}
$$

From $\sum_{m=0}^{n-R} \frac{\left(\frac{C}{\alpha}\right)^{m}}{m !} \leqslant e^{\frac{C}{\alpha}}$ and Corollary 21 , it follows that $\mathcal{R}_{n} \in \mathcal{O}\left(\Gamma_{\beta+2}^{\alpha}(n-R)\right)$, because $\left(\begin{array}{l}l-1 \\ l-R\end{array}\right)$ is a polynomial in $l$. Therefore, for all $R \in \mathbb{N}_{0}$

$$
\begin{aligned}
& {\left[x^{n}\right] f\left(g^{-1}(x)\right)=\sum_{m=0}^{n-R}\left(\begin{array}{c}
n+\beta+1 \\
m
\end{array}\right) \sum_{k=0}^{R-1} c_{k, m} \Gamma_{\beta+2}^{\alpha}(n-m-k)+\mathcal{O}\left(\Gamma_{\beta+2}^{\alpha}(n-R)\right) } \\
= & \sum_{k=0}^{R-1}\left[x^{k}\right]\left(\mathcal{A}_{\beta+2}^{\alpha} B_{\beta}(x) \sum_{m=0}^{n-R}\left(\begin{array}{c}
n+\beta+1 \\
m
\end{array}\right) A(x)^{m} \Gamma_{\beta+2}^{\alpha}(n-m-k)\right)+\mathcal{O}\left(\Gamma_{\beta+2}^{\alpha}(n-R)\right),
\end{aligned}
$$

where $\mathcal{A}_{\beta+2}^{\alpha}$ acts on everything on its right. Applying Corollary 45 with $x \rightarrow A(x)$ to the inner sum and reordering result in the statement.

Lemma 50. If $\alpha, \beta \in \mathbb{R}_{>0}, R \in \mathbb{N}_{0}$ and $A, B_{\gamma}$ as defined in eqs. (29) and (32), then

$$
\left[x^{n}\right] f\left(g^{-1}(x)\right)=\sum_{k=0}^{R-1} \sum_{l=0}^{R-1-k} c_{k, l}^{\prime}\left(\begin{array}{c}
l+k-1 \\
l
\end{array}\right) \Gamma_{\beta+2}^{\alpha}(n-l-k)+\mathcal{O}\left(\Gamma_{\beta+2}^{\alpha}(n-R)\right) \quad \forall R \in \mathbb{N}_{0},
$$

where $c_{k, l}^{\prime}:=\left[x^{k}\right]\left(\mathcal{A}_{\beta+2}^{\alpha} B_{\beta}(x) A(x)^{l} e^{\frac{A(x)}{\alpha}}\right)(x)$. 
Proof. Set $c_{k, l, m}$ as in Lemma 49. By Lemma 42 there exists a constant $C \in \mathbb{R}$ such that $\rho_{\beta+2, R}^{\alpha}\left(B_{\beta}(x) A(x)^{l+m}\right) \leqslant C^{l+m+1}$ for all $l, m \in \mathbb{N}_{0}$. It follows from the second part of Observation 17 that

$$
\left|c_{k, l, m}\right|=\frac{\alpha^{-m}}{m !}\left|\left[x^{k}\right]\left(\mathcal{A}_{\beta+2}^{\alpha} B_{\beta}(x) A(x)^{l+m}\right)(x)\right| \leqslant \frac{\alpha^{-m}}{m !} C^{l+m+1} \quad \forall k, l, m \in \mathbb{N}_{0} \text { with } k \leqslant R .
$$

Therefore, for all $k \leqslant R$ and $n \geqslant 2 R-k$,

$$
\begin{aligned}
& \left|\sum_{l=R-k}^{n-R} \sum_{m=0}^{n-R-l} c_{k, l, m}\left(\begin{array}{c}
l+k-1 \\
l
\end{array}\right) \Gamma_{\beta+2}^{\alpha}(n-l-k)\right| \\
\leqslant & \sum_{l=R-k}^{n-R} \sum_{m=0}^{n-R-l} \frac{\alpha^{-m} C^{l+m+1}}{m !}\left(\begin{array}{c}
l+k-1 \\
l
\end{array}\right) \Gamma_{\beta+2}^{\alpha}(n-l-k)
\end{aligned}
$$

which is in $\mathcal{O}\left(\Gamma_{\beta+2}^{\alpha}(n-R)\right)$, because $\sum_{m=0}^{n-R-l} \frac{\alpha^{-m} C^{m}}{m !} \leqslant e^{\frac{C}{\alpha}}$ and by Corollary 21. Applying this to truncate the summation over $l$ in eq. (40) from Lemma 49 gives for all $R \in \mathbb{N}_{0}$

$$
\left[x^{n}\right] f\left(g^{-1}(x)\right)=\sum_{k=0}^{R-1} \sum_{l=0}^{R-k-1} \sum_{m=0}^{n-R-l} c_{k, l, m}\left(\begin{array}{c}
l+k-1 \\
l
\end{array}\right) \Gamma_{\beta+2}^{\alpha}(n-l-k)+\mathcal{O}\left(\Gamma_{\beta+2}^{\alpha}(n-R)\right) .
$$

Note that $\left(\begin{array}{c}n+m \\ n\end{array}\right) \geqslant 1 \Rightarrow(n+m) ! \geqslant n ! m !$ and therefore

$$
\sum_{m=n}^{\infty} \frac{C^{m}}{m !}=\sum_{m=0}^{\infty} \frac{C^{n+m}}{(n+m) !} \leqslant \frac{C^{n}}{n !} \sum_{m=0}^{\infty} \frac{C^{m}}{m !}=e^{C} \frac{C^{n}}{n !} .
$$

It follows from this and eq. (43) that for all $n \geqslant R-l+1$ and $k+l \leqslant R$

$$
\begin{gathered}
\left|\sum_{m=n-R-l+1}^{\infty} c_{k, l, m} \Gamma_{\beta+2}^{\alpha}(n-l-k)\right| \leqslant C^{l+1} \sum_{m=n-R-l+1}^{\infty} \frac{\left(\frac{C}{\alpha}\right)^{m}}{m !} \Gamma_{\beta+2}^{\alpha}(n-l-k) \\
\leqslant e^{\frac{C}{\alpha}} C^{l+1}\left(\frac{C}{\alpha}\right)^{n-l-R+1} \frac{\Gamma_{\beta+2}^{\alpha}(n-l-k)}{(n-R-l+1) !}
\end{gathered}
$$

which is in $\mathcal{O}\left(\Gamma_{\beta+2}^{\alpha}(n-R)\right)$ as long as $k$ and $l$ are bounded, because $\frac{\Gamma(n-l-k+\beta+2)}{\Gamma(n-R-l+2)} \sim$ $n^{R-k+\beta}$. Applying this to complete the summation over $m$ in eq. (44) and noting that $c_{k, l}^{\prime}=\sum_{m=0}^{\infty} c_{k, l, m}$ results in eq. (42).

Corollary 51. If $\alpha, \beta \in \mathbb{R}_{>0}, R \in \mathbb{N}_{0}$ and $A, B_{\gamma}$ as defined in eqs. (29) and (32), then $f \circ g^{-1} \in \mathbb{R}[[x]]_{\beta+2}^{\alpha}$ and

$$
\left[x^{k}\right]\left(\mathcal{A}_{\beta+2}^{\alpha} f \circ g^{-1}\right)(x)=\left[x^{k}\right]\left(\mathcal{A}_{\beta+2}^{\alpha} B_{\beta-k+1}(x) e^{\frac{A(x)}{\alpha}}\right)(x) \quad \forall k \in \mathbb{N}_{0} .
$$


Proof. After the change of summation variables $k \rightarrow k+l$, eq. (42) becomes

$$
\left[x^{n}\right] f\left(g^{-1}(x)\right)=\sum_{k=0}^{R-1} \sum_{l=0}^{k} c_{k-l, l}^{\prime}\left(\begin{array}{c}
k-1 \\
l
\end{array}\right) \Gamma_{\beta+2}^{\alpha}(n-k)+\mathcal{O}\left(\Gamma_{\beta+2}^{\alpha}(n-R)\right) \quad \forall R \in \mathbb{N}_{0} .
$$

By Definition 1, this equation states that $f \circ g^{-1} \in \mathbb{R}[[x]]_{\beta+2}^{\alpha}$ and that the coefficients of the asymptotic expansion are

$$
\begin{gathered}
c_{k}^{f \circ g^{-1}}=\sum_{l=0}^{k} c_{k-l, l}^{\prime}\left(\begin{array}{c}
k-1 \\
l
\end{array}\right)=\sum_{l=0}^{k}\left[x^{k-l}\right]\left(\mathcal{A}_{\beta+2}^{\alpha} B_{\beta}(x) A(x)^{l}\left(\begin{array}{c}
k-1 \\
l
\end{array}\right) e^{\frac{A(x)}{\alpha}}\right)(x) \\
=\left[x^{k}\right] \sum_{l=0}^{\infty} x^{l}\left(\mathcal{A}_{\beta+2}^{\alpha} B_{\beta}(x) A(x)^{l}\left(\begin{array}{c}
k-1 \\
l
\end{array}\right) e^{\frac{A(x)}{\alpha}}\right)(x) \\
=\left[x^{k}\right]\left(\mathcal{A}_{\beta+2}^{\alpha} B_{\beta}(x) \sum_{l=0}^{\infty}(x A(x))^{l}\left(\begin{array}{c}
k-1 \\
l
\end{array}\right) e^{\frac{A(x)}{\alpha}}\right)(x),
\end{gathered}
$$

where $x^{l}\left(\mathcal{A}_{\beta+2}^{\alpha} f(x)\right)(x)=\left(\mathcal{A}_{\beta+2}^{\alpha} x^{l} f(x)\right)(x)$ for all $f \in \mathbb{R}[[x]]_{\beta+2}^{\alpha}$ was used, which follows from the product rule (Proposition 22). Because of $\sum_{l=0}^{\infty}\left(\begin{array}{c}k-1 \\ l\end{array}\right)(x A(x))^{l}=(1+$ $x A(x))^{k-1}=\left(\frac{x}{g(x)}\right)^{k-1}$ and the definition of $B_{\gamma}$ in Lemma 41, the statement follows.

Proof of Theorem 35. Because of Lemma 37, we may assume that $\beta \in \mathbb{R}_{>0}$ and start with the expression from Corollary 51 for $\left[x^{k}\right]\left(\mathcal{A}_{\beta+2}^{\alpha} f \circ g^{-1}\right)(x)$. We will use Lemmas 40 and 41 to expand this expression. By Corollary 51 and the product rule (Proposition 22), we have for all $k \in \mathbb{N}_{0}$

$$
\left[x^{k}\right]\left(\mathcal{A}_{\beta+2}^{\alpha} f \circ g^{-1}\right)(x)=\left[x^{k}\right]\left(e^{\frac{A(x)}{\alpha}}\left(\mathcal{A}_{\beta+2}^{\alpha} B_{\beta-k+1}\right)(x)+B_{\beta-k+1}(x)\left(\mathcal{A}_{\beta+2}^{\alpha} e^{\frac{A(x)}{\alpha}}\right)(x)\right) .
$$

Applying Lemma 41 on the first term of this expression gives after a straightforward but lengthy calculation,

$$
\begin{gathered}
{\left[x^{k}\right] e^{\frac{A(x)}{\alpha}}\left(\mathcal{A}_{\beta+2}^{\alpha} B_{\beta-k+1}\right)(x)=\left[x^{k}\right] e^{\frac{A(x)}{\alpha}}\left(\frac{g(x)}{x}\right)^{\beta-k+1}\left(x^{2} g^{\prime}(x)\left(\mathcal{A}_{\beta}^{\alpha} f\right)(x)\right.} \\
\left.+f(x)\left(x(\beta-k+1) g^{\prime}(x) \frac{x}{g(x)}+\alpha^{-1}-\beta x+x^{2} \frac{\partial}{\partial x}\right)\left(\mathcal{A}_{\beta}^{\alpha} g\right)(x)\right) \\
=\left[x^{k}\right] e^{\frac{A(x)}{\alpha}}\left(\frac{g(x)}{x}\right)^{\beta-k+1}\left(x^{2} g^{\prime}(x)\left(\mathcal{A}_{\beta}^{\alpha} f\right)(x)\right. \\
\left.+\left(-x^{2} f^{\prime}(x)+\alpha^{-1} f(x) g^{\prime}(x)\left(\frac{x}{g(x)}\right)^{2}\right)\left(\mathcal{A}_{\beta}^{\alpha} g\right)(x)\right)
\end{gathered}
$$


where the identity $\left[x^{k}\right] x p^{\prime}(x) q(x)=k\left[x^{k}\right] p(x) q(x)-\left[x^{k}\right] x p(x) q^{\prime}(x)$ for all $p, q \in \mathbb{R}[[x]]$ was used to eliminate the summand which contains the $\frac{\partial}{\partial x}\left(\mathcal{A}_{\beta}^{\alpha} g\right)(x)$ factor. By Lemma 40 , the second term on the right hand side of eq. (46) is

$$
\begin{gathered}
{\left[x^{k}\right] B_{\beta-k+1}(x)\left(\mathcal{A}_{\beta+2}^{\alpha} e^{\frac{A(x)}{\alpha}}\right)(x)=-\left[x^{k}\right] \alpha^{-1} B_{\beta-k+1}(x)\left(\frac{x}{g(x)}\right)^{2} e^{\frac{A(x)}{\alpha}}\left(\mathcal{A}_{\beta}^{\alpha} g\right)(x)} \\
=-\left[x^{k}\right] \alpha^{-1} f(x) g^{\prime}(x)\left(\frac{g(x)}{x}\right)^{\beta-k+1}\left(\frac{x}{g(x)}\right)^{2} e^{\frac{A(x)}{\alpha}}\left(\mathcal{A}_{\beta}^{\alpha} g\right)(x),
\end{gathered}
$$

where the definition of $B_{\beta-k+1}(x)$ from Lemma 41 was substituted. Summing both expressions for the terms in eq. (46) from eqs. (47) and (48) and substituting the definition of $A(x)$ from Lemma 40 results in

$$
\left[x^{k}\right]\left(\mathcal{A}_{\beta+2}^{\alpha} f \circ g^{-1}\right)(x)=\left[x^{k}\right] x^{2} e^{\frac{\frac{1}{g(x)}-\frac{1}{x}}{\alpha}}\left(\frac{g(x)}{x}\right)^{\beta-k+1}\left(g^{\prime}(x)\left(\mathcal{A}_{\beta}^{\alpha} f\right)(x)-f^{\prime}(x)\left(\mathcal{A}_{\beta}^{\alpha} g\right)(x)\right),
$$

for all $k \in \mathbb{N}_{0}$. By Proposition 14, the $x^{2}$ prefactor indicates that $f \circ g^{-1}$ is actually in the subspace $\mathbb{R}[[x]]_{\beta}^{\alpha} \subset \mathbb{R}[[x]]_{\beta+2}^{\alpha}$ and

$$
\left[x^{k}\right]\left(\mathcal{A}_{\beta}^{\alpha} f \circ g^{-1}\right)(x)=\left[x^{k}\right] e^{\frac{\frac{1}{g(x)}-\frac{1}{x}}{\alpha}}\left(\frac{g(x)}{x}\right)^{\beta-k-1}\left(g^{\prime}(x)\left(\mathcal{A}_{\beta}^{\alpha} f\right)(x)-f^{\prime}(x)\left(\mathcal{A}_{\beta}^{\alpha} g\right)(x)\right) .
$$

If we set $p(x):=e^{\frac{\frac{1}{g(x)}-\frac{1}{x}}{\alpha}}\left(\frac{g(x)}{x}\right)^{\beta}\left(\left(\mathcal{A}_{\beta}^{\alpha} f\right)(x)-\frac{f^{\prime}(x)}{g^{\prime}(x)}\left(\mathcal{A}_{\beta}^{\alpha} g\right)(x)\right)$ and $q(x):=g(x)$, we obtain

$$
\left[x^{k}\right]\left(\mathcal{A}_{\beta}^{\alpha} f \circ g^{-1}\right)(x)=\left[x^{k}\right] p(x) q^{\prime}(x)\left(\frac{x}{q(x)}\right)^{k+1}=\left[x^{k}\right] p\left(q^{-1}(x)\right) \quad \forall k \in \mathbb{N}_{0},
$$

by Lemma 46. After replacing $p$ and $q$ by their expressions, we obtain

$$
\left(\mathcal{A}_{\beta}^{\alpha} f \circ g^{-1}\right)(x)=e^{\frac{\frac{1}{x-\frac{1}{g^{-1}(x)}}}{\alpha}}\left(\frac{x}{g^{-1}(x)}\right)^{\beta}\left(\left(\mathcal{A}_{\beta}^{\alpha} f\right)\left(g^{-1}(x)\right)-\frac{f^{\prime}\left(g^{-1}(x)\right)}{g^{\prime}\left(g^{-1}(x)\right)}\left(\mathcal{A}_{\beta}^{\alpha} g\right)\left(g^{-1}(x)\right)\right) .
$$

The special case $f(x)=x$ with an application of the identity $g^{\prime}\left(g^{-1}(x)\right)=\frac{1}{\left(g^{-1}\right)^{\prime}(x)}$ results in eq. (26). Solving eq. (26) for $\left(\mathcal{A}_{\beta}^{\alpha} g\right)\left(g^{-1}(x)\right)$ and substituting the result into eq. (49) gives eq. (25) with the substitution $g \rightarrow g^{-1}$.

Remark 52. Bender and Richmond [9] established that $\left[x^{n}\right](1+g(x))^{\gamma n+\delta}=n \gamma e^{\frac{\gamma g_{1}}{\alpha}} g_{n}+$ $\mathcal{O}\left(g_{n}\right)$ if $g_{n} \sim \alpha n g_{n-1}$ and $g_{0}=0$. Using Lagrange inversion, the first coefficient in the expansion of the compositional inverse in eq. (26) can be obtained from this. In this respect, Theorem 35 is a generalization of Bender and Richmond's result.

In the same article Bender and Richmond proved a theorem similar to Theorem 35 for the class of power series $f$ whose coefficients grow more rapidly than factorially such that $n f_{n-1} \in o\left(f_{n}\right)$. Theorem 35 establishes a link to the excluded case $n f_{n-1}=\mathcal{O}\left(f_{n}\right)$. 
Remark 53. The restriction $g_{1}=1$ ensures that our power series actually have compositional inverses and that we do not leave the ring $\mathbb{R}[[x]]_{\beta}^{\alpha}$. We might also allow a non-zero positive value for $g_{1}$. To do this it is sufficient to allow composition with the family of power series $h_{\gamma}(x)=\gamma x \in \mathbb{R}[[x]]$ where $\gamma \in \mathbb{R}_{>0}$. Right composition with $h_{\gamma}, f \mapsto f \circ h_{\gamma}$ is a trivial isomorphisms of vector spaces $\mathbb{R}[[x]]_{\beta}^{\alpha} \rightarrow \mathbb{R}[[x]]_{\beta}^{\gamma \alpha}$, which follows immediately from Definition 1. Every power series $g(x) \in \mathbb{R}[[x]]_{\beta}^{\alpha}$ with $g_{0}=0$ and $g_{1}>0$ can be decomposed into $g=\widetilde{g} \circ h_{g_{1}}$ such that $\widetilde{g}_{1}=1$ and $\widetilde{g} \in \mathbb{R}[[x]]_{\beta}^{\alpha / g_{1}}$. The asymptotics of the coefficients of the composition $f \circ g=f \circ \widetilde{g} \circ h_{g_{1}}$ can be calculated using Theorem 35 if $f, \widetilde{g} \in \mathbb{R}[[x]]_{\beta}^{\alpha^{\prime}}$ for some $\alpha^{\prime} \geqslant \frac{\alpha}{g_{1}}$.

Remark 54. The chain rule in eq. (25) exposes a peculiar algebraic structure. It would be useful to have a combinatorial interpretation of the $e^{\frac{\frac{1}{x}-\frac{1}{g(x)}}{\alpha}}$ term.

\section{Some remarks on differential equations}

Differential equations arising from physical systems form an active field of research in the scope of resurgence $[21,3]$. Unfortunately, the exact calculation of an overall factor of the asymptotic expansion of a solution of an ODE, called Stokes constant, turns out to be difficult for many problems. This fact severely limits the utility of the method for enumeration problems, as the dominant factor of the asymptotic expansion is of most interest and the detailed structure of the asymptotic expansion is secondary.

In this section it will be sketched, for the sake of completeness, how the presented combinatorial framework fits into the realm of differential equations. The given elementary properties each have their counterpart in resurgence's alien calculus [25, II.6].

Corollary 25 serves as a good starting point to analyze differential equations with power series solutions in $\mathbb{R}[[x]]_{\beta}^{\alpha}$. Given a polynomial $F \in \mathbb{R}\left[x, y_{0}, \ldots, y_{L}\right]$, the $\mathcal{A}_{\beta}^{\alpha}$-derivation can be applied to the ordinary differential equation

$$
0=F\left(x, f(x), f^{\prime}(x), f^{\prime \prime}(x), \ldots, f^{(L)}(x)\right) .
$$

Applying the $\mathcal{A}$-derivation naively to both sides of this equation and using the chain rule for the composition with polynomials results in a linear equation for the asymptotic expansions of the derivatives $f^{(l)}$. Proposition 38 tells us, how the asymptotic expansions of the $f^{(l)}$ relate to each other. We will follow this line of thought in detail in

Proposition 55. If $F \in \mathbb{R}\left[x, y_{0}, \ldots, y_{L}\right]$ and $f \in \mathbb{R}[[x]]_{\beta}^{\alpha}$ is a solution of the differential equation

$$
0=F\left(x, f(x), f^{\prime}(x), f^{\prime \prime}(x), \ldots, f^{(L)}(x)\right)
$$

then $\left(\mathcal{A}_{\beta}^{\alpha} f\right)(x)$ is a solution of the linear differential equation

$$
0=\sum_{l=0}^{L} x^{2 L-2 l} \frac{\partial F}{\partial y_{l}}\left(x, f^{(0)}, \ldots, f^{(L)}\right)\left(\prod_{j=0}^{l-1}\left(\alpha^{-1}-x(\beta+2 j)+x^{2} \frac{\partial}{\partial x}\right)\right)\left(\mathcal{A}_{\beta}^{\alpha} f\right)(x)
$$


Proof. From Proposition 38, Proposition 14 and $f \in \mathbb{R}[[x]]_{\beta}^{\alpha}$, it follows that $f^{(l)} \in$ $\mathbb{R}[[x]]_{\beta+2 l}^{\alpha} \subset \mathbb{R}[[x]]_{\beta+2 L}^{\alpha}$ for all $L \geqslant l$. By Corollary 25 , we can apply $\mathcal{A}_{\beta+2 L}^{\alpha}$ to both sides of eq. (50) and use Proposition 14,

$$
\begin{aligned}
0 & =\sum_{l=0}^{L} \frac{\partial F}{\partial y_{l}}\left(x, f^{(0)}, \ldots, f^{(L)}\right)\left(\mathcal{A}_{\beta+2 L}^{\alpha} f^{(l)}\right)(x) \\
& =\sum_{l=0}^{L} \frac{\partial F}{\partial y_{l}}\left(x, f^{(0)}, \ldots, f^{(L)}\right) x^{2(L-l)}\left(\mathcal{A}_{\beta+2 l}^{\alpha} f^{(l)}\right)(x) .
\end{aligned}
$$

Iterating Proposition 38 gives

$$
\begin{aligned}
\left(\mathcal{A}_{\beta+2 l}^{\alpha} f^{(l)}\right)(x) & =\left(\alpha^{-1}-x(\beta+2(l-1))+x^{2} \frac{\partial}{\partial x}\right)\left(\mathcal{A}_{\beta+2(l-1)}^{\alpha} f^{(l-1)}\right)(x) \\
& =\left(\prod_{j=0}^{l-1}\left(\alpha^{-1}-x(\beta+2 j)+x^{2} \frac{\partial}{\partial x}\right)\right)\left(\mathcal{A}_{\beta}^{\alpha} f\right)(x) .
\end{aligned}
$$

Substituting this into eq. (52) results in eq. (51).

Remark 56. Even if it is known that the coefficients of the power series solution of a differential equation have a well-behaved asymptotic expansion, Proposition 55 provides this asymptotic expansion only up to the initial values for the linear differential equation (51). Note that the form of the asymptotic expansion can still depend non-trivially on the initial values of the solution $f$ of a non-linear differential equation.

Remark 57. The linear differential equation (51) only has a non-trivial solution in $\mathbb{R}[[x]]$ if $\alpha^{-1}$ is the root of a certain polynomial. More specifically, making a power series ansatz for $\left(\mathcal{A}_{\beta}^{\alpha} f\right)(x)$ in eq. (51) gives

$$
0=\left[x^{m}\right] \sum_{l=0}^{L} x^{2 L-2 l} \alpha^{-l} \frac{\partial F}{\partial y_{l}}\left(x, f^{(0)}, \ldots, f^{(L)}\right),
$$

where $m$ is the smallest integer such that the equation is not trivially fulfilled. If this root is not real or if two roots have the same modulus, the present formalism has to be generalized to complex and multiple $\alpha$ to express the asymptotic expansion of a general solution. This generalization is straightforward. We merely need to generalize Definition 1 of suitable sequences to:

Definition 58. For given $\beta \in \mathbb{R}$ and $\alpha_{1}, \ldots, \alpha_{L} \in \mathbb{C}$ with $\left|\alpha_{1}\right|=\left|\alpha_{2}\right|=\ldots=\left|\alpha_{L}\right|=$ : $\alpha>0$ let $\mathbb{C}[[x]]_{\beta}^{\alpha_{1}, \ldots, \alpha_{L}} \subset \mathbb{C}[[x]]$ be the subspace of complex power series, such that $f \in \mathbb{C}[[x]]_{\beta}^{\alpha_{1}, \ldots, \alpha_{L}}$ if and only if there exist sequences of complex numbers $\left(c_{k, l}^{f}\right)_{k \in \mathbb{N}_{0}, l \in[1, L]}$, which fulfill

$$
f_{n}=\sum_{k=0}^{R-1} \sum_{l=1}^{L} c_{k, l}^{f} \Gamma_{\beta}^{\alpha_{l}}(n-k)+\mathcal{O}\left(\Gamma_{\beta}^{\alpha}(n-k)\right) \quad \forall R \in \mathbb{N}_{0} .
$$




\section{Applications}

\subsection{Connected chord diagrams}

A chord diagram with $n$ chords is a circle with $2 n$ points, which are labeled by integers $1, \ldots, 2 n$ and connected in disjoint pairs by $n$ chords. There are $(2 n-1) !$ ! such diagrams.

A chord diagram is connected if no set of chords can be separated from the remaining chords by a line which does not cross any chords. Let $I(x)=\sum_{n=0}(2 n-1) ! ! x^{n}$, the ordinary generating function of all chord diagrams, and $C(x)=\sum_{n=0} C_{n} x^{n}$, where $C_{n}$ is the number of connected chord diagrams with $n$ chords. Following [19], the power series $I(x)$ and $C(x)$ are related by,

$$
I(x)=1+C\left(x I(x)^{2}\right)
$$

This functional equation can be solved for the coefficients of $C(x)$ by basic iterative methods. The first coefficients are

$$
C(x)=x+x^{2}+4 x^{3}+27 x^{4}+248 x^{5}+\ldots
$$

This sequence is entry A000699 in Neil Sloane's integer sequence on-line encyclopedia [26].

Because $(2 n-1) ! !=\frac{2^{n+\frac{1}{2}}}{\sqrt{2 \pi}} \Gamma\left(n+\frac{1}{2}\right)=\frac{1}{\sqrt{2 \pi}} \Gamma_{\frac{1}{2}}^{2}(n)$, the power series $I$ is in $\mathbb{R}[[x]]_{\frac{1}{2}}^{2}$ and $\left(\mathcal{A}_{\frac{1}{2}}^{2} I\right)(x)=\frac{1}{\sqrt{2 \pi}}$ as a direct consequence of Definitions 1 and 8. From eq. (54), it also follows that $C\left(x I(x)^{2}\right) \in \mathbb{R}[[x]]_{\frac{1}{2}}^{2}$. Because $x I(x)^{2} \in \mathbb{R}[[x]]_{\frac{1}{2}}^{2}$ by the product rule (Proposition 22), we know from Corollary 36 with $f(x)=C(x)$ and $g(x)=x I(x)^{2}$ that $C \in \mathbb{R}[[x]]_{\frac{1}{2}}^{2}$.

Applications of the general chain rule from Theorem 35 and the product rule on the functional eq. (54) result in

$$
\begin{gathered}
\left(\mathcal{A}_{\frac{1}{2}}^{2} I\right)(x)=\left(\mathcal{A}_{\frac{1}{2}}^{2}\left(1+C\left(x I(x)^{2}\right)\right)\right)(x)=\left(\mathcal{A}_{\frac{1}{2}}^{2} C\left(x I(x)^{2}\right)\right)(x) \\
=2 x I(x) C^{\prime}\left(x I(x)^{2}\right)\left(\mathcal{A}_{\frac{1}{2}}^{2} I\right)(x)+\left(\frac{x}{x I(x)^{2}}\right)^{\frac{1}{2}} e^{\frac{x I(x)^{2}-x}{2 x^{2} I(x)^{2}}}\left(\mathcal{A}_{\frac{1}{2}}^{2} C\right)\left(x I(x)^{2}\right) .
\end{gathered}
$$

which can be solved for $\left(\mathcal{A}_{\frac{1}{2}}^{2} C\right)\left(x I(x)^{2}\right)$,

$$
\left(\mathcal{A}_{\frac{1}{2}}^{2} C\right)\left(x I(x)^{2}\right)=\frac{I(x)-2 x I(x)^{2} C^{\prime}\left(x I(x)^{2}\right)}{\sqrt{2 \pi}} e^{\frac{1-I(x)^{2}}{2 x I(x)^{2}}},
$$

where $\left(\mathcal{A}_{\frac{1}{2}}^{2} I\right)(x)=\frac{1}{\sqrt{2 \pi}}$ was used. This can be composed with the unique $y \in \mathbb{R}[[x]]$ which solves $y(x) I(y(x))^{2}=x$,

$$
\left(\mathcal{A}_{\frac{1}{2}}^{2} C\right)(x)=\frac{I(y(x))-2 x C^{\prime}(x)}{\sqrt{2 \pi}} e^{\frac{1-I(y(x))^{2}}{2 x}} .
$$




\begin{tabular}{|c||c||c|c|c|c|c|c|c|c|}
\hline sequence & 0 & 1 & 2 & 3 & 4 & 5 & 6 & 7 & 8 \\
\hline \hline$\frac{\sqrt{2 \pi}}{e^{-1}}\left(\mathcal{A}_{\frac{1}{2}}^{2} C\right)$ & 1 & $-\frac{5}{2}$ & $-\frac{43}{8}$ & $-\frac{579}{16}$ & $-\frac{44477}{128}$ & $-\frac{5326191}{1280}$ & $-\frac{180306541}{3072}$ & $-\frac{203331297947}{215040}$ & $-\frac{58726239094693}{3440640}$ \\
\hline$\frac{\sqrt{2 \pi}}{e^{-1}}\left(\mathcal{A}_{\frac{1}{2}}^{2} M\right)$ & 1 & -4 & -6 & $-\frac{154}{3}$ & $-\frac{1610}{3}$ & $-\frac{34588}{5}$ & $-\frac{4666292}{45}$ & $-\frac{553625626}{315}$ & $-\frac{1158735422}{35}$ \\
\hline
\end{tabular}

Table 1: First coefficients of the asymptotic expansions of $C_{n}$ and $M_{n}$.

From eq. (54), it follows that $I(y(x))=1+C(x)$, therefore

$$
\left(\mathcal{A}_{\frac{1}{2}}^{2} C\right)(x)=\frac{1+C(x)-2 x C^{\prime}(x)}{\sqrt{2 \pi}} e^{-\frac{1}{2 x}\left(2 C(x)+C(x)^{2}\right)} .
$$

It can be verified, using the closed form of its coefficients, that the power series $I(x)$ fulfills the differential equation $2 x^{2} I^{\prime}(x)+x I(x)+1=I(x)$. From this and eq. (54), the non-linear differential equation $C^{\prime}(x)=\frac{C(x)(1+C(x))-x}{2 x C(x)}$ [19] for $C(x)$ can be deduced. Using this on the expression for $\left(\mathcal{A}_{\frac{1}{2}}^{2} C\right)(x)$ from eq. (57) results in the simplification,

$$
\left(\mathcal{A}_{\frac{1}{2}}^{2} C\right)(x)=\frac{1}{\sqrt{2 \pi}} \frac{x}{C(x)} e^{-\frac{1}{2 x}\left(2 C(x)+C(x)^{2}\right)} .
$$

This is the generating function of the full asymptotic expansion of $C_{n}$. The first coefficients are,

$$
\left(\mathcal{A}_{\frac{1}{2}}^{2} C\right)(x)=\frac{e^{-1}}{\sqrt{2 \pi}}\left(1-\frac{5}{2} x-\frac{43}{8} x^{2}-\frac{579}{16} x^{3}-\frac{44477}{128} x^{4}-\frac{5326191}{1280} x^{5}+\ldots\right) .
$$

By Definitions 1 and 8 as well as $\frac{1}{\sqrt{2 \pi}} \Gamma_{\frac{1}{2}}^{2}(n)=(2 n-1)$ !!, we get the two equivalent expressions for the asymptotic expansion of the coefficients $C_{n}$ :

$$
\begin{array}{ll}
C_{n}=\sum_{k=0}^{R-1} \Gamma_{\frac{1}{2}}^{2}(n-k)\left[x^{k}\right]\left(\mathcal{A}_{\frac{1}{2}}^{2} C\right)(x)+\mathcal{O}\left(\Gamma_{\frac{1}{2}}^{2}(n-R)\right) & \forall R \in \mathbb{N}_{0} \\
C_{n}=\sqrt{2 \pi} \sum_{k=0}^{R-1}(2(n-k)-1) ! !\left[x^{k}\right]\left(\mathcal{A}_{\frac{1}{2}}^{2} C\right)(x)+\mathcal{O}((2(n-R)-1) ! !) & \forall R \in \mathbb{N}_{0} .
\end{array}
$$

The first terms of this large $n$ expansion are

$$
C_{n}=e^{-1}\left((2 n-1) ! !-\frac{5}{2}(2 n-3) ! !-\frac{43}{8}(2 n-5) ! !-\frac{579}{16}(2 n-7) ! !+\ldots\right) .
$$

The first term, $e^{-1}$, of this expansion has been computed by Kleitman [23], Stein and Everett [27] and Bender and Richmond [9] each using different methods. With the presented method an arbitrary number of coefficients can be computed. Some additional coefficients are given in Table 1.

The probability of a random chord diagram with $n$ chords to be connected is therefore $e^{-1}\left(1-\frac{5}{4 n}\right)+\mathcal{O}\left(\frac{1}{n^{2}}\right)$. 


\subsection{Monolithic chord diagrams}

A chord diagram is called monolithic if it consists only of a connected component and of isolated chords which do not 'contain' each other [19]. That means with $(a, b)$ and $(c, d)$ the labels of two chords, it is not allowed that $a<c<d<b$ nor $c<a<b<d$. Let $M(x)=\sum_{n=0} M_{n} x^{n}$ be the generating function of monolithic chord diagrams. Following [19], $M(x)$ fulfills

$$
M(x)=C\left(\frac{x}{(1-x)^{2}}\right)
$$

Clearly, Theorem 35 implies that $M \in \mathbb{R}[[x]]_{\frac{1}{2}}^{2}$, because $C \in \mathbb{R}[[x]]_{\frac{1}{2}}^{2}$ and $\frac{x}{(1-x)^{2}} \in \mathbb{R}\{x\} \subset$ $\mathbb{R}[[x]]_{\frac{1}{2}}^{2}$. Using the $\mathcal{A}_{\frac{1}{2}}^{2}$-derivation on both sides of this equation together with the result for $\left(\mathcal{A}_{\frac{1}{2}}^{2} C\right)(x)$ in eq. (58) gives

$$
\begin{aligned}
\left(\mathcal{A}_{\frac{1}{2}}^{2} M\right)(x) & =\frac{1}{\sqrt{2 \pi}} \frac{1}{(1-x)} \frac{x}{M(x)} e^{1-\frac{x}{2}-\frac{(1-x)^{2}}{2 x}\left(2 M(x)+M(x)^{2}\right)} \\
& =\frac{1}{\sqrt{2 \pi}}\left(1-4 x-6 x^{2}-\frac{154}{3} x^{3}-\frac{1610}{3} x^{4}-\frac{34588}{5} x^{5}+\ldots\right) .
\end{aligned}
$$

Some additional coefficients are given in Table 1 . The probability of a random chord diagram with $n$ chords to be non-monolithic is therefore $1-\left(1-\frac{4}{2 n-1}+\mathcal{O}\left(\frac{1}{n^{2}}\right)\right)=\frac{2}{n}+$ $\mathcal{O}\left(\frac{1}{n^{2}}\right)$.

\subsection{Simple permutations}

A permutation is called simple if it does not map a non-trivial interval to another interval. Expressed formally, the permutation $\pi \in S_{n}^{\text {simple }} \subset S_{n}$ if and only if $\pi([i, j]) \neq[k, l]$ for all $i, j, k, l \in[1, n]$ with $2 \leqslant|[i, j]| \leqslant n-1$. See Albert, Atkinson and Klazar [1] for a detailed exposition of simple permutations. Set $S(x)=\sum_{n=4}^{\infty}\left|S_{n}^{\text {simple }}\right| x^{n}$, the generating function of simple permutations ${ }^{2}$, and $F(x)=\sum_{n=1}^{\infty} n ! x^{n}$, the generating function of all permutations. Following [1], $S(x)$ and $F(x)$ are related by the equation

$$
\frac{F(x)-F(x)^{2}}{1+F(x)}=x+S(F(x)),
$$

which can be solved iteratively for the coefficients of $S(x)$ :

$$
S(x)=2 x^{4}+6 x^{5}+46 x^{6}+338 x^{7}+2926 x^{8}+\cdots
$$

This sequence is entry A111111 of the OEIS [26] with the different convention, A111111= $x+2 x^{2}+S(x)$.

\footnotetext{
${ }^{2}$ We adopt the convention of Albert, Atkinson and Klazar and do not consider permutations below order 4 as simple.
} 
As $n !=\Gamma_{1}^{1}(n), F(x) \in \mathbb{R}[[x]]_{1}^{1}$ and $\left(\mathcal{A}_{1}^{1} F\right)=1$ by Definitions 1 and 8 . Therefore, the full asymptotic expansion of $S(x)$ can be obtained by applying the general chain rule to both sides of eq. (62). Alternatively, eq. (62) implies $\frac{x-x^{2}}{1+x}=F^{-1}(x)+S(x)$ with $F^{-1}(F(x))=x$. By Theorem 35, it follows from $F \in \mathbb{R}[[x]]_{1}^{1}, F_{0}=0$ and $F_{1}=1$ that $F^{-1} \in \mathbb{R}[[x]]_{1}^{1}$. By linearity and $\frac{x-x^{2}}{1+x} \in \mathbb{R}\{x\} \subset \mathbb{R}[[x]]_{1}^{1}$, we also have $S \in \mathbb{R}[[x]]_{1}^{1}$. The expression for the asymptotic expansion of $F^{-1}(x)$ in terms of $\left(\mathcal{A}_{1}^{1} F\right)(x)$ from eq. (26) gives

$$
\left(\mathcal{A}_{1}^{1} S\right)(x)=\left(\mathcal{A}_{1}^{1} \frac{x-x^{2}}{1+x}\right)(x)-\left(\mathcal{A}_{1}^{1} F^{-1}\right)(x)=\left(F^{-1}\right)^{\prime}(x) \frac{x}{F^{-1}(x)} e^{\frac{1}{x}-\frac{1}{F^{-1}(x)}},
$$

where $\frac{x-x^{2}}{1+x} \in \operatorname{ker} \mathcal{A}_{1}^{1}$ was used. Observe that $F(x)$ fulfills the differential equation $x^{2} F^{\prime}(x)+(x-1) F(x)+x=0$, from which a non-linear differential equation for $F^{-1}(x)$ can be deduced, because $F^{\prime}\left(F^{-1}(x)\right)\left(F^{-1}\right)^{\prime}(x)=1$ :

$$
\left(F^{-1}\right)^{\prime}(x)=\frac{1}{F^{\prime}\left(F^{-1}(x)\right)}=\frac{F^{-1}(x)^{2}}{\left(1-F^{-1}(x)\right) x-F^{-1}(x)} .
$$

Using this together with $\frac{x-x^{2}}{1+x}=F^{-1}(x)+S(x)$ gives

$$
\left(\mathcal{A}_{1}^{1} S\right)(x)=\frac{x F^{-1}(x)}{x-(1+x) F^{-1}(x)} e^{\frac{1}{x}-\frac{1}{F^{-1}(x)}}=\frac{1}{1+x} \frac{1-x-(1+x) \frac{S(x)}{x}}{1+(1+x) \frac{S(x)}{x^{2}}} e^{-\frac{2+(1+x) \frac{S(x)}{x^{2}}}{1-x-(1+x) \frac{S(x)}{x}}} .
$$

The coefficients of $\left(\mathcal{A}_{1}^{1} S\right)(x)$ can be computed iteratively. The first coefficients are

$$
\left(\mathcal{A}_{1}^{1} S\right)(x)=e^{-2}\left(1-4 x+2 x^{2}-\frac{40}{3} x^{3}-\frac{182}{3} x^{4}-\frac{7624}{15} x^{5}+\cdots\right) .
$$

By Definitions 1 and 8, this is an expression for the asymptotics of the number of simple permutations

$$
\left|S_{n}^{\text {simple }}\right|=\sum_{k=0}^{R-1}(n-k) !\left[x^{k}\right]\left(\mathcal{A}_{1}^{1} S\right)(x)+\mathcal{O}((n-R) !) \quad \forall R \in \mathbb{N}_{0} .
$$

Therefore, the asymptotic expansion starts with

$$
\left|S_{n}^{\text {simple }}\right|=e^{-2}\left(n !-4(n-1) !+2(n-2) !-\frac{40}{3}(n-3) !-\frac{182}{3}(n-4) !+\cdots\right) .
$$

Albert, Atkinson and Klazar [1] calculated the first three terms of this expansion. With the presented methods the calculation of the asymptotic expansions $\left(\mathcal{A}_{1}^{1} S\right)(x)$ or $\left(\mathcal{A}_{1}^{1} F^{-1}\right)(x)$ up to order $n$ is as easy as calculating the expansion of $S(x)$ or $F^{-1}(x)$ up to order $n+2$. Some additional coefficients are given in Table 2. 


\begin{tabular}{|c||c||c|c|c|c|c|c|c|c|c|}
\hline sequence & 0 & 1 & 2 & 3 & 4 & 5 & 6 & 7 & 8 & 9 \\
\hline \hline$\frac{1}{e^{-2}}\left(\mathcal{A}_{1}^{1} S\right)$ & 1 & -4 & 2 & $-\frac{40}{3}$ & $-\frac{182}{3}$ & $-\frac{7624}{15}$ & $-\frac{202652}{45}$ & $-\frac{14115088}{315}$ & $-\frac{30800534}{63}$ & $-\frac{16435427656}{2835}$ \\
\hline
\end{tabular}

Table 2: First coefficients of the asymptotic expansion of $\left|S_{n}^{\text {simple }}\right|$.

Remark 59. The examples above are chosen to demonstrate that given a (functional) equation which relates two power series in $\mathbb{R}[[x]]_{\beta}^{\alpha}$, it is often an easy task to calculate the full asymptotic expansion of one of the power series from the asymptotic expansion of the other power series. Applications include functional equations for 'irreducible combinatorial objects'. The two examples fall into this category. Irreducible combinatorial objects were studied in general by Beissinger [4].

Remark 60. Eqs. (58), (61) and (65) expose another interesting algebraic property. Proposition 15 and the chain rule imply that $\left(\mathcal{A}_{\frac{1}{2}}^{2} C\right)(x) \in \mathbb{R}[[x]]_{\frac{3}{2}}^{2},\left(\mathcal{A}_{\frac{1}{2}}^{2} M\right)(x) \in \mathbb{R}[[x]]_{\frac{3}{2}}^{2}$ and $\left(\mathcal{A}_{1}^{1} S\right)(x) \in \mathbb{R}[[x]]_{3}^{1}$. This way, the 'higher-order' asymptotics of the asymptotic sequence can be calculated by iterating the application of the $\mathcal{A}$ map. With resurgence, it might be possible to construct convergent large-order expansions for these cases. The fact that the asymptotics of each sequence may be expressed as a combination of polynomial and exponential expressions of the original sequence can be seen as an avatar of resurgence.

Remark 61. In quantum field theory the coupling, an expansion parameter, needs to be reparametrized in the process of renormalization [15]. Those reparametrizations are merely compositions of power series which are believed to be Gevrey-1. Theorem 35 might be useful for the resummation of renormalized quantities in quantum field theory. DysonSchwinger equations in quantum field theory can be stated as functional equations of a form similar to the above $[14,11]$. These considerations were the subject of the publication [13], where the presented formalism was applied to zero-dimensional quantum field theory and the enumeration of graphs.

\section{Acknowledgements}

Many thanks to Dirk Kreimer for steady encouragement and counseling. I wish to express my gratitude to David Broadhurst. He sparked my interest in asymptotic expansions and I benefited greatly from our discussions. I wish to thank Inês Aniceto who patiently introduced me to the basics of resurgence theory, David Sauzin for encouragement and patiently clarifying details of the connections to resurgence, Julien Courtiel for helpful comments regarding the closure properties of $\mathbb{R}[[x]]_{\beta}^{\alpha}$ under functional inversion and Karen Yeats for hospitality during the revision of this article. I also wish to thank the anonymous referee for carefully reading the manuscript and providing many helpful comments.

\section{References}

[1] MH Albert, MD Atkinson, and M Klazar. The enumeration of simple permutations. Journal of Integer Sequences, volume 6:03.4.4, 2003. 
[2] G Alvarez. Langer-Cherry derivation of the multi-instanton expansion for the symmetric double well. Journal of mathematical physics, 45(8):3095-3108, 2004.

[3] I Aniceto, R Schiappa, and M Vonk. The resurgence of instantons in string theory. Communications in Number Theory and Physics, 6(2):339-496, 2012.

[4] JS Beissinger. The enumeration of irreducible combinatorial objects. Journal of Combinatorial Theory, Series A, 38(2):143-169, 1985.

[5] CM Bender and TT Wu. Anharmonic oscillator. Physical Review, 184(5):1231-1260, 1969.

[6] EA Bender. Asymptotic methods in enumeration. SIAM Review, 16(4):485-515, 1974.

[7] EA Bender. An asymptotic expansion for the coefficients of some formal power series. Journal of the London Mathematical Society, 2(3):451-458, 1975.

[8] EA Bender and ER Canfield. The asymptotic number of labeled graphs with given degree sequences. Journal of Combinatorial Theory, Series A, 24(3):296-307, 1978.

[9] EA Bender and LB Richmond. An asymptotic expansion for the coefficients of some power series II: Lagrange inversion. Discrete Mathematics, 50:135-141, 1984.

[10] F Bergeron, G Labelle, and P Leroux. Combinatorial species and tree-like structures, volume 67. Cambridge University Press, 1998.

[11] M Borinsky. Algebraic lattices in qft renormalization. Letters in Mathematical Physics, 106(7):879-911, 2016.

[12] M Borinsky. Generating asymptotics for factorially divergent sequences (extended abstract). In Séminaire Lotharingien de Combinatoire - Proceedings to FPSAC 2017, volume 78B, page 12pp., 2017.

[13] M Borinsky. Renormalized asymptotic enumeration of Feynman diagrams. Annals of Physics, 385:95-135, 2017.

[14] DJ Broadhurst and D Kreimer. Exact solutions of Dyson-Schwinger equations for iterated one-loop integrals and propagator-coupling duality. Nuclear Physics B, 600(2):403-422, 2001.

[15] A Connes and D Kreimer. Renormalization in quantum field theory and the Riemann-Hilbert problem II: The $\beta$-function, diffeomorphisms and the renormalization group. Communications in Mathematical Physics, 216(1):215-241, 2001.

[16] NG de Bruijn. Asymptotic Methods in Analysis. Bibliotheca mathematica. Dover Publications, 1970.

[17] GV Dunne and M Ünsal. Resurgence and trans-series in quantum field theory: the $\mathbb{C P}^{N-1}$ model. Journal of High Energy Physics, 2012(11):1-86, 2012.

[18] J Écalle. Les fonctions résurgentes. Publ. math. d'Orsay/Univ. de Paris, Dep. de math., 1981.

[19] P Flajolet and M Noy. Analytic combinatorics of chord diagrams. In Formal Power Series and Algebraic Combinatorics, pages 191-201. Springer, 2000. 
[20] P Flajolet and R Sedgewick. Analytic combinatorics. Cambridge University Press, 2009.

[21] S Garoufalidis, A Its, A Kapaev, and M Marino. Asymptotics of the instantons of Painlevé I. International Mathematics Research Notices, 2012(3):561-606, 2012.

[22] PF Hsieh and Y Sibuya. Basic Theory of Ordinary Differential Equations. Universitext. Springer New York, 2012.

[23] DJ Kleitman. Proportions of irreducible diagrams. Studies in Applied Mathematics, 49(3):297-299, 1970.

[24] J Le Guillou and J Zinn-Justin. Large-order behaviour of perturbation theory, volume 7. Elsevier, 2012.

[25] C Mitschi and D Sauzin. Divergent Series, Summability and Resurgence I. Springer, 2016.

[26] NJA Sloane. The on-line encyclopedia of integer sequences. http://oeis .org/, 2005.

[27] PR Stein and CJ Everett. On a class of linked diagrams II. asymptotics. Discrete Mathematics, 21(3):309-318, 1978.

[28] HS Wilf. generatingfunctionology. Elsevier, 2013. 